\title{
Crowdsource Based Indoor Localization by Uncalibrated Heterogeneous Wi-Fi Devices
}

\author{
Wooseong Kim, ${ }^{1}$ Sungwon Yang, ${ }^{2}$ Mario Gerla, ${ }^{2}$ and Eun-Kyu Lee ${ }^{3}$ \\ ${ }^{1}$ Department of Computer Engineering, Gachon University, 1342 Seongnam-ro, Gyeonggi 461701, Republic of Korea \\ ${ }^{2}$ Department of Computer Science, University of California, Los Angeles, Los Angeles, CA 90095, USA \\ ${ }^{3}$ Department of Information and Telecommunication Engineering, Incheon National University, 119 Academy-ro, \\ Yeonsu-gu, Incheon 406772, Republic of Korea
}

Correspondence should be addressed to Eun-Kyu Lee; eklee@cs.ucla.edu

Received 15 March 2016; Accepted 4 May 2016

Academic Editor: Sergio F. Ochoa

Copyright (C) 2016 Wooseong Kim et al. This is an open access article distributed under the Creative Commons Attribution License, which permits unrestricted use, distribution, and reproduction in any medium, provided the original work is properly cited.

\begin{abstract}
Many indoor localization techniques that rely on received signals from Wi-Fi access points have been explored in the last decade. Recently, crowdsourced Wi-Fi fingerprint attracts much attention, which leads to a self-organized localization system avoiding painful survey efforts. However, this participatory approach introduces new challenges with no previously proposed techniques such as heterogeneous devices, short measurement time, and multiple values for a single position. This paper proposes an efficient localization method combating the three major technical issues in the crowdsourcing based systems. We evaluate our indoor positioning method using 5 places with different radio environment and 8 different mobile phones. The experimental results show that the proposed approach provides consistent localization accuracy and outperforms existing localization algorithms.
\end{abstract}

\section{Introduction}

Navigation ability of smart phones and tablets at indoor environment becomes a challenge as recent mobile Location Based Services (LBSs) [1-3] require more accurate and seamless positioning at both indoor and outdoor environment. Outdoor localization had been provided reasonably by global satellite positioning systems using Global Positioning System (GPS), GLObal NAvigation Satellite System (GLONASS), Galileo, and so forth. On contrary, the indoor localization still continues to a challenge even though it has been exploited during last several years. Several techniques for the indoor localization have been proposed [4-6]. In a survey of indoor localization techniques [7], many techniques are compared such as GPS, RFID, WLAN, and Bluetooth in terms of accuracy, complexity, and scalability. Some of previous works uses a specially designed hardware like a beacon installed on walls or ceilings such as RFID, ultrasound, and infrared technologies [8-10]. Other systems use a combination of different sensors to increase accuracy $[11,12]$. Those which use the hardware of short range communication technologies are costly to deploy on a large area, even though those systems provide fine-grained accuracy in indoor localization.

In contrast, Wi-Fi based positioning systems are very common and easily achieved as shown that Internet map services of Google, Apple, Microsoft, and so forth already use received signals from many deployed access points (APs) for localization. Recently, Wi-Fi is becoming more popular and ubiquitous with countless Wi-Fi-enabled smart devices. Accordingly, indoor localization using the Wi-Fi APs receives much attention from mobile computing research area, which has the advantage of avoiding the cost of specialized hardware deployment.

Typically, Wi-Fi based localization estimates the location based on observed and stored "fingerprints" which are composed of a MAC address and corresponding Received Signal Strength (RSS) value from a Wi-Fi AP $[10,13]$. While previous researches mainly dealt with localization algorithms to improve accuracy based on the fingerprint database, recent studies focus on building the database with less effort. "Crowdsource" Based Indoor Localization (CIL) [14-19] by autonomous users is one of cost efficient approaches, which 
reduces the map building and maintenance expense compared to an expert surveyor based approach. The CIL is driven by normal mobile users who participate in the $\mathrm{Wi}$ Fi AP survey as a contributor, a user, or both. Also, this multiple-surveyor-multiple-user model has the advantages of fast radio map building and quick update.

Figure 1 shows an example of the CIL, which consists of two phases: "training phase" (or "offline phase") and "localization phase" (or "online phase"). In the training phase, fingerprint data of locations have been captured to establish a radio map. After then, mobile phones inquire their location with measured fingerprint data to a remote server that holds the radio map in the localization phase. In the figure, the five users (i.e., A, B, C, D, and E) are untrained normal people who are carrying different types of mobile phones. Users A, D, and E are surveyors for Room 101, Room 102, and Room 103 who measure RSS of Wi-Fi APs and upload the measured data with corresponding AP information to a local DB server. The uploaded data is processed on the server and converted to fingerprint data. When user B inquires the current location information, the server returns to Room 101 if user B's RSS measurement matches one from the user A.

However, the CIL also introduces a new set of challenges. First, the CIL has to extract accurate fingerprint values from short measurement time for Received Signal Strength (RSS) because the mobile users as voluntary surveyors probably provide short-term RSS measurements. Second, the CIL has to support calibration-free indoor localization across different devices. Typically, users as surveyors carry various types of devices in terms of hardware and software, for example, Wi-Fi chipsets, antenna pattern, and operating systems. The RSS measurement results in different values across the heterogeneous devices even at exactly the same positions.

This crowdsourcing approach has been considered in many recent researches [14-19]. However, many of them address those challenges in the CIL. In [20] about surveyfree localization, several researches [21-23] dealt with heterogeneous devices for CIL using difference of RSS samples from APs instead of absolute RSS values to combat measurement variance from heterogeneous devices. Previously, we proposed "Freeloc" [24] that uses a list of APs ordered by RSS for the fingerprint instead of the RSS itself. Thus, the Freeloc consistently results in robust and reliable outcomes resolving those challenges. In this paper, we compare the Freeloc with existing practical indoor localization algorithms for the CIL in terms of accuracy. In addition, we investigate how to decide a gap between sample RSSs for difference localization environment and conduct measurement study for the Freeloc in variance of wireless channel because of interference from dense Wi-Fi APs, deep fading from many pedestrians, and path loss from short distance.

The remainder of this paper is organized as follows: we introduce background knowledge with related works on indoor localization schemes in Section 2. Section 3 introduces existing localization algorithms for CIL. Section 4 describes our system and algorithm together with the three major challenges covered in the CIL. We show the performance evaluation and comparison with existing localization algorithms from Sections 5 to 7. In Section 5, Freeloc

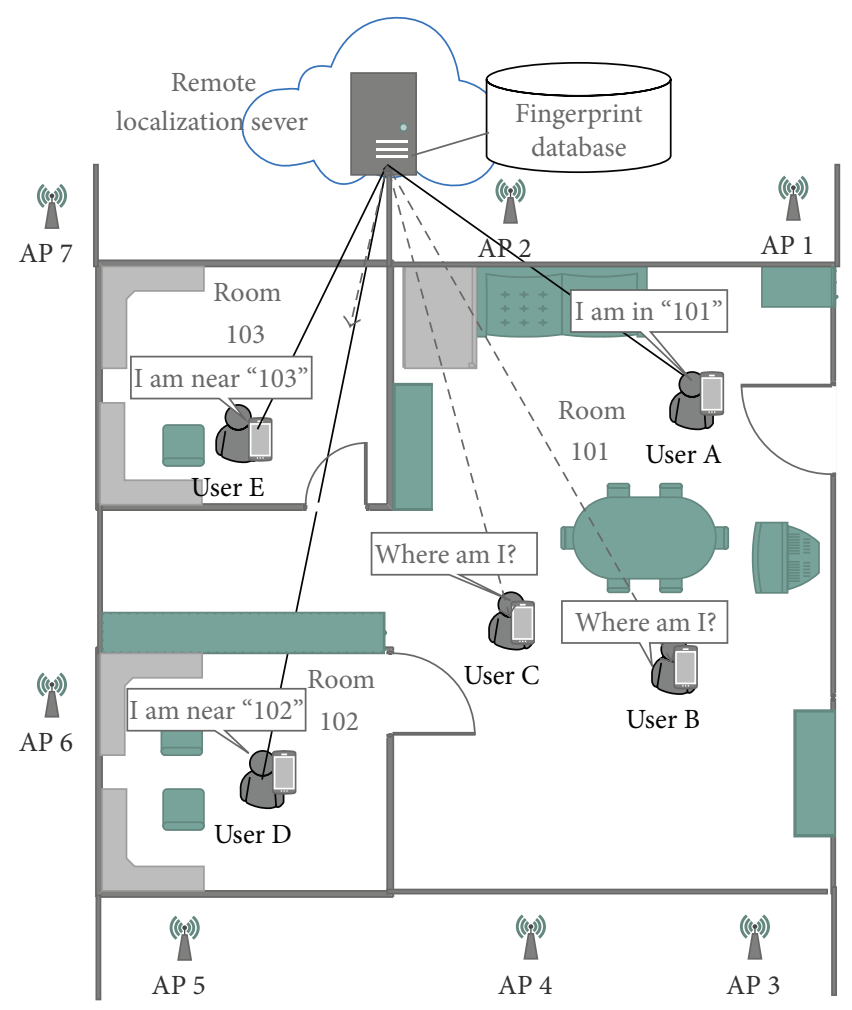

FIGURE 1: Crowdsource Based Indoor Localization (CIL) approach which collects RSS values of Wi-Fi APs measured by multiple users, users A-E at different positions of Rooms 101, 102, and 103, together with corresponding AP information, which is finally converted to fingerprint data of each location.

performance is compared according to various localization environment. We compare Freeloc with other CIL algorithms in Section 6. In Section 7, Freeloc performance is investigated with varying delta values. We conclude the paper in Section 8 .

\section{Background}

2.1. Indoor Localization Techniques. Many indoor localization techniques have been proposed over the past decade. Some indoor localization techniques require special hardware to determine the devices location like active badge [9] and active bat [25] that are attached on ceilings and walls of buildings and transmits out infrared and ultrasound pulses for proximity estimation. The cricket and cricket compass [8, 26] used a combination of RF and ultrasound technologies. Techniques using active RFID were also proposed [27, 28].

Recently, Wi-Fi based indoor localization receives much attention as Wi-Fi APs become used for outdoor localization. Like the outdoor localization in cellular networks, triangulation using time difference of signal arrival from neighbour APs can be considered $[13,29]$. This time of arrival (TOA) based approach could suffer from timely varying signal path at complex indoor environment. Instead, Received Signal Strength (RSS) can be used. RADAR [10] adopts this RF signal intensity first for the purpose of indoor localization. Now, many researchers concentrate on techniques that use Wi-Fi 
RSS data. Studies like [30] analyzed the properties of Received Signal Strength values reported by wireless network interface cards. References $[5,31]$ show that the Wi-Fi fingerprint (i.e., a basic service set identification (BSSID) and its RSS value) methods can fulfil accuracy required for many indoor LBS applications.

2.2. Crowdsourced Indoor Localization. Survey-free localization [16-19] or Crowdsource Based Indoor Localization (CIL) $[14,15]$ has rigorously been explored in order to reduce effort and cost involved in expert surveyor based system as smart hand-held devices are deployed widely. The CIL is a selforganized participatory system where normal mobile users contribute measurement of Wi-Fi fingerprints at various locations for localization. Reference [15] carried out experiments in indoor environments and have discussed encouraging results. Teller et al. in [16] have designed and deployed an organic location system and achieved position accuracy that is comparable to the accuracy achieved by a survey driven system. A crowdsourcing based approach by Ledlie et al. models the world as a tree of hierarchical namespaces and provides an algorithm that explicitly accounts for temporal variations in signal space [19]. Recently, accelerator assisted dead-reckoning techniques are adopted for enhancing accuracy of crowdsourced indoor localization [11, 12].

A main issue with the CIL is the heterogeneity of hardware devices, usually a variety of mobile phones, to collect Wi-Fi fingerprints during the training phase. This leads to variation in the values of observed signal strength measurements due to the different chipsets present on different devices. Park et al. explore this issue in [17] and compare various methods used to mitigate this problem. The main parameters that are used are signal strength values and access point detection. The Kernel function based estimation, which predicts user location using a naive Bayes classifier, has been proposed to obtain better results than linear transformation based approaches [32]. Tsui et al. proposed an unsupervised learning method that automatically tries to solve hardware variance problem in Wi-Fi localization [33]. Wu et al. proposed site survey-free indoor localization with off-the-shelf devices, which needs complex conversion from virtual to physical space based on measurement [21]. References [2224] show that robust fingerprints can be derived using signal strength difference (SSD), instead of absolute RSS values in the heterogeneous mobile devices for measurement.

\section{Localization Algorithm for CIL}

Although several localization algorithms were proposed for CIL, most of them use log-distance or similarity of RSSs [20]. We introduce several practical approaches that are less complicated and easy to apply for the fingerprint based indoor localization to compare performance with Freeloc.

First, Euclidean distance of RSSs of $k$-nearest neighbours $(k-\mathrm{NN})$ is calculated to find a location with minimum value of summation of the distance values. Here we consider unweighted $k$-NN in which distance values are equally added at the summation. Second, similarity based on the Tanimoto coefficient is considered. Third, we evaluate the $N$-gram that compares BSSID subsequences between two fingerprints to calculate probability of appearance of the same contiguous subsequence. With these four algorithms, we apply the same experiment environment that is used for "Freeloc" evaluation for the comparison:

(i) $k$-nearest-neighbour $(k-\mathrm{NN})$ is a popular localization method to analyze RSS pattern from $k$ neighbour APs using some recognition techniques. For instance, it can simply calculate Euclidean distance of two fingerprints' RSS values that are measured and stored in a remote server. Like this, $k$-NN estimates a location with the distance values of most frequently appeared $k$ neighbour APs:

$$
\arg \min _{f_{L_{i}} \in F} \sqrt{\sum_{i=1} k\left\|f_{i}-f_{L_{i}}\right\|}
$$

where $L_{i}$ is locations $\left\{L_{0}, L_{1}, \ldots, L_{n}\right\}$ and $f_{i}$ is a measured fingerprint and $f_{L_{i}}$ is a stored fingerprint of location $i$. That is to say, $k$-NN selects a location that has minimum sum of distance of most strong $k$ fingerprints between the measured and stored values.

(ii) Two fingerprint similarities can be evaluated by Tanimoto coefficient, which is widely used for estimating similarity of Wi-Fi fingerprints. Reference [34] shows that place detection can be achieved by similarity of radio environment using Tanimoto coefficient. As in below equation, location $L_{i}$ with maximum Tanimoto coefficient can be chosen:

$$
\arg \max _{f_{L_{i}} \in F} T\left(f_{i}, f_{L_{i}}\right)=\frac{f_{i} \cdot f_{L_{i}}}{\left\|f_{i}\right\|^{2}+\left\|f_{L_{i}}\right\|^{2}-f_{i} \cdot f_{L_{i}}} \text {. }
$$

(iii) $N$-gram algorithm is a different approach compared to above two approaches, which calculates likelihood of two subsequences of the measured and stored fingerprints [35]. $N$-gram only concerns strength order of the fingerprints while $k-\mathrm{NN}$ and Tanimoto considers signal strength itself. The order of fingerprint strength does not change probably even if the signal strength of the fingerprints changes dynamically. For this, $N$-gram sorts the fingerprints in descending order of the RSS and compares subsequences of the fingerprints. For instance, subsequence $N$-gram $\left(f_{s}\right)=\left\{f_{s}, f_{s+1}, \ldots, f_{s+n-1}\right\}$ is a fingerprint sequence from a sth fingerprint to a $i+(n-1)$ th fingerprint with size of $n$. Here we can find a location, $L_{i}$ that maximizes a following equation:

$$
\arg \max _{f_{L_{i}} \in F} \prod_{s} P\left(N-\operatorname{gram}\left(f_{s}\right) \mid L_{i}\right) P\left(L_{i}\right) .
$$

That is to say, the algorithm selects a location with maximum probability that a set of the subsequences $f_{s}$ appears in the location $L_{i}$. N-gram motivates us to improve it for crowdsourced localization in which measured fingerprints are varying with locations and devices as described in a following subsection. 


\section{Freeloc: Calibration-Free Indoor Localization}

In this section, we briefly describe our localization algorithm, Freeloc, with a usage scenario in Figure 1. Freeloc solves following three critical challenges in fingerprint derivation based on the RSSs captured by untrained mobile users:

(i) RSS measurement for short duration: it is well known that multipath fading in an indoor environment causes RSS to fluctuate over time even if the receiver is absolutely fixed [36]. One simple method to reduce RSS variance is to record the RSS data for a long time. As the number of RSS samples increases it is easier to identify one single fingerprint value which ensures construction of a more robust and accurate radio map. However, it is almost impossible to have normal users collect RSS data for such a long time in the crowdsourcing based model. We believe that the RSS survey time at each location will not exceed one minute assuming all the surveyors are usual mobile users. Therefore, a technique that extracts accurate fingerprint data from short-time measurement is necessary.

(ii) Device heterogeneity in CIL system: it is inevitable that diverse devices get involved in establishing the radio map database. Since there is no expert surveyor who uses specially designed hardware, it is highly likely that the RSS data gathered from each user varies even though it is collected at the exact same position. Different chipsets and antenna designs of the Wi-Fi devices cause varying RSS records per each device and make it difficult to calibrate them. This device heterogeneity is another key problem for crowdsourced localization.

(iii) Multiple measurements for one location: another problem is that more than one user can upload one's own fingerprint data with the same location label, but it is obtained at different measurement points. This results in multiple fingerprint data sets for one location, which leads to inefficient memory usage and more time to estimate the current location. None of the previous studies take this into account even though this is also a principal cause of localization accuracy degradation.

4.1. Fingerprint Value Extraction. This section presents how a RSS value of each fingerprint can be extracted at a particular location with variation in response rate and RSS values. In contrast RSS can be measured for a long time (e.g., from more than one hour to a month) to get average value in the expert surveyor system [37]; CIL has short measurement duration to extract robust fingerprint. We propose a practical and resilient RSS abstraction method for the fingerprint based on the experimental results we conducted:

(i) AP response rate: in our experiments, we observed strong correlation between AP response rate and RSS. Ledlie et al. [19] thought that the correlation between the response rate and RSS is rather weak, which led to using the response rate as fingerprint information with RSS. However, our results show that APs with RSS values of greater than $-70 \mathrm{dBm}$ provide over $90 \%$ of response rate and APs with RSS between $-70 \mathrm{dBm}$ and $-85 \mathrm{dBm}$ provide over $50 \%$ of response rate. APs with RSS of less than $-90 \mathrm{dBm}$ present very poor response rate. Therefore, we decide to give lower weights to weak RSS values, which reflects the lower AP response rate naturally for fingerprint.

(ii) RSS variance: RSS of a particular AP is varying over time due to shadowing and multipath fading, and RSS fluctuation could be higher in indoor environment rather than outdoor, which degrades the localization accuracy. For CIL that requires short measurement duration, probabilistic methods based on Gaussian distribution were proposed in several studies proposed $[16,19,32]$ to obtain the average value, which are however feasible only in ideal environment.

Instead, we propose a simple yet effective method based on our experiment results that show the most frequently captured RSS in short duration is very close to the most RSS in the long-duration measurement case. In other words, most frequent RSSs can be obtained regardless of the duration of measurement time. The proposed method extracts fingerprint RSS values by ignoring RSS records that are far away from the most frequent RSS and giving the maximum weight to the RSSs with peak value as shown in (4), which provides tolerance to the RSS variation over time. $V_{\mathrm{fp}}$ is the fingerprint value for an AP and RSS $\mathrm{Seak}_{\text {p }}$ is the RSS value of highest frequency during the measurement. The width of the range is set by $\omega_{L T}$ and $\omega_{R T}$ :

$$
\begin{aligned}
& V_{\mathrm{fp}} \\
& =\frac{\sum_{n=1}^{\omega_{L T}}\left(\mathrm{RSS}_{\text {peak }}-n\right)+\mathrm{RSS}_{\text {peak }}+\sum_{m=1}^{\omega_{R T}}\left(\mathrm{RSS}_{\text {peak }}+m\right)}{\omega_{L T}+\omega_{R T}+1} .
\end{aligned}
$$

\subsection{Online Localization Algorithm}

4.2.1. Relative RSS Comparison. Most of previous localization techniques estimate a location with an absolute RSS value based on the radio map built during the offline phase. However, CIL where surveyors carry heterogeneous hardware devices can have different fingerprint data sets even for the same location because RSS values measured by those devices are different from each other.

In this section, we explain a novel localization technique, Freeloc, instead of modifying existing techniques for the heterogeneous device environment that is aligned with our target indoor usage scenarios. Thanks to the proliferation of Wi-Fi technology, some office or university buildings have more than 50 APs installed for personal purpose or for offloading of wireless cellular networks. RSS values of those APs are widely distributed (e.g., $-40 \mathrm{dBm}$ to $-100 \mathrm{dBm}$ ), which is good to abstract relative RSS strength among APs. 


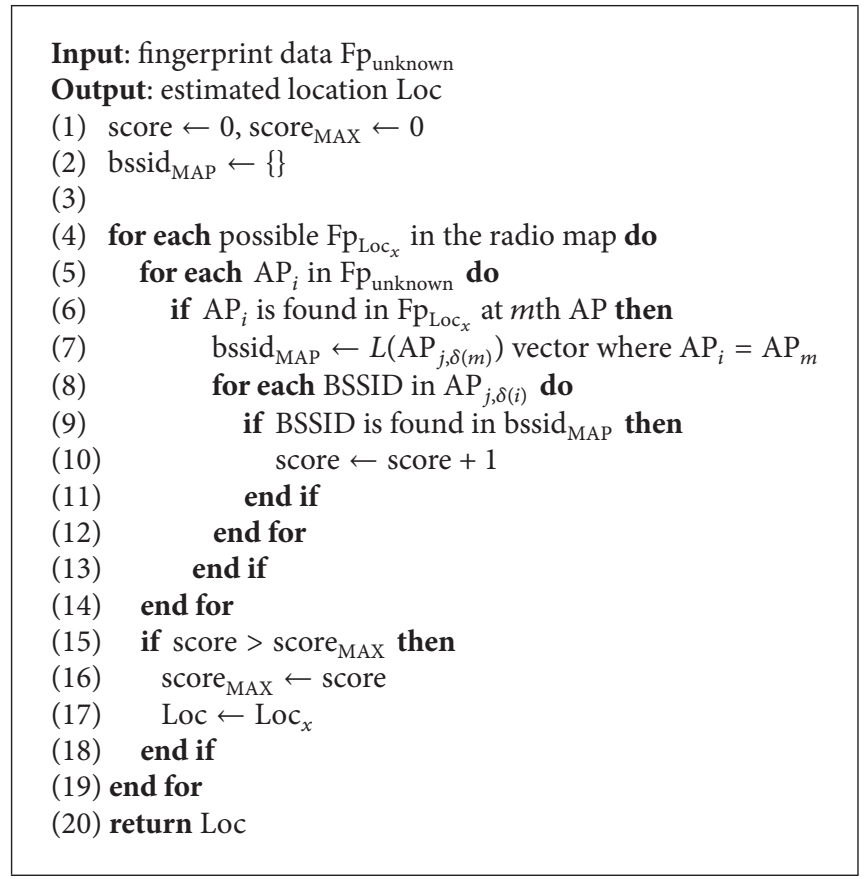

Algorithm 1: Estimate the location.

TABLE 1: RSS measurement results from each user.

\begin{tabular}{lcccc}
\hline AP & User A & User B & User C & User D \\
\hline AP1 & -50 & -65 & -65 & -92 \\
AP2 & -55 & -68 & -51 & -80 \\
AP3 & -67 & -74 & -53 & -67 \\
AP4 & -72 & -52 & -66 & -75 \\
AP5 & -88 & -91 & -82 & -58 \\
AP6 & -90 & -87 & -85 & -54 \\
\hline
\end{tabular}

In contrast to several approaches using RSS difference of APs for fingerprints, Freeloc generates fingerprints using an AP order instead of RSS value itself as shown in

$$
\begin{array}{r}
\operatorname{Fp}\left(\operatorname{Loc}_{x}\right)=\left\{\operatorname{Loc}_{x},\left[\operatorname{AP}_{i}, L\left(\operatorname{AP}_{j, \delta(i)}\right)\right]\right\} \\
i, j \in \operatorname{APs} \text { of } \operatorname{Loc}_{x}, i \neq j
\end{array}
$$

$$
\operatorname{RSS}\left(\mathrm{AP}_{i}\right)-\operatorname{RSS}\left(\mathrm{AP}_{j}\right) \geq \delta \text {. }
$$

Each fingerprint data element Fp of location $\operatorname{Loc}_{x}$ has a location label $\operatorname{Loc}_{x}$ (e.g., room number) where measurement is taken, and a detected AP's BSSID $\left(\mathrm{AP}_{i}\right)$ that has the $i$ th strongest $V_{\mathrm{fp}} . L\left(\mathrm{AP}_{j, \delta}\right)$ is an order of other detected APs' BSSIDs of which RSS is weaker than RSS of the $\mathrm{AP}_{i}$ by more than $\delta$ value. Our proposed method adopts the delta $(\delta)$ as a marginal value for varying RSS which is different from $N$-gram [35] that considers only similarity between two continuous subsequences of the ordered fingerprint even though both schemes focus on relationship between detected APs in terms of RSS, rather than RSS value itself. The delta value keeps the relationship consistent under wireless

\begin{tabular}{|c|c|}
\hline \multicolumn{2}{|c|}{ Room 101} \\
\hline $\mathrm{AP}_{i}$ & $\mathrm{AP}_{j, \delta(i)}$ \\
\hline $\mathrm{AP}^{*}$ & $\{\mathrm{AP} 3, \mathrm{AP} 4, \mathrm{AP} 5, \mathrm{AP} 6\}$ \\
\hline $\mathrm{AP} 2^{*}$ & $\{\mathrm{AP} 3, \mathrm{AP} 4, \mathrm{AP} 5, \mathrm{AP} 6\}$ \\
\hline AP3 & $\{$ AP5, AP6 $\}$ \\
\hline AP4 & $\{$ AP5, AP6 $\}$ \\
\hline AP5 & \{\} \\
\hline AP6 & \{\} \\
\hline
\end{tabular}

TABLE 2: Fingerprint data of each location.

(a)

(b)

\begin{tabular}{lcc} 
& Room 102 & \\
AP $_{i}$ & $\mathrm{AP}_{j, \delta(i)}$ \\
\hline AP6 $^{*}$ & $\{\mathrm{AP} 1, \mathrm{AP} 2, \mathrm{AP} 3, \mathrm{AP} 4\}$ \\
$\mathrm{AP5}^{*}$ & $\{\mathrm{AP} 1, \mathrm{AP} 2, \mathrm{AP} 4\}$ \\
$\mathrm{AP} 3$ & $\{\mathrm{AP} 1, \mathrm{AP} 2\}$ \\
$\mathrm{AP} 4$ & $\{\mathrm{AP} 1\}$ \\
$\mathrm{AP} 2$ & $\{\mathrm{AP} 1\}$ \\
$\mathrm{AP} 1$ & \{\} \\
\hline
\end{tabular}

channel fading and enables our method to deal with heterogeneous devices without calibration efforts and also multiple Fp elements for the same location from many different devices.

For example, suppose that users A and D are the surveyors and users B and $\mathrm{C}$ want to know their positions in Figure 1, and the measurement of user $\mathrm{A}-\mathrm{D}$ is presented in Table 1; the fingerprints of Rooms 101 and 102 can be defined in Table 2. For instance, AP1 with $-50 \mathrm{dBm}$ RSS value has 4 


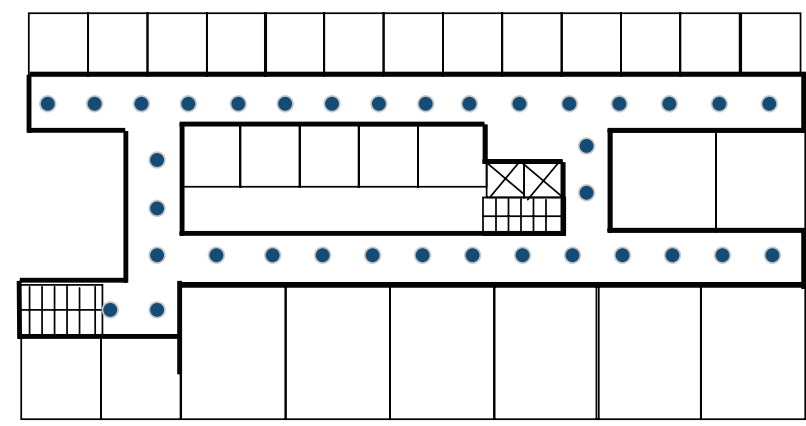

(a) 5th floor of Gachon IT building

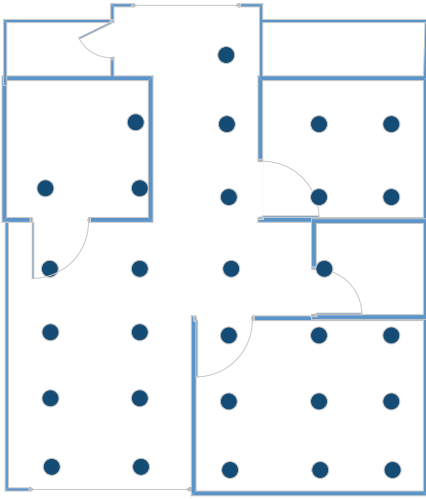

(c) Resident house

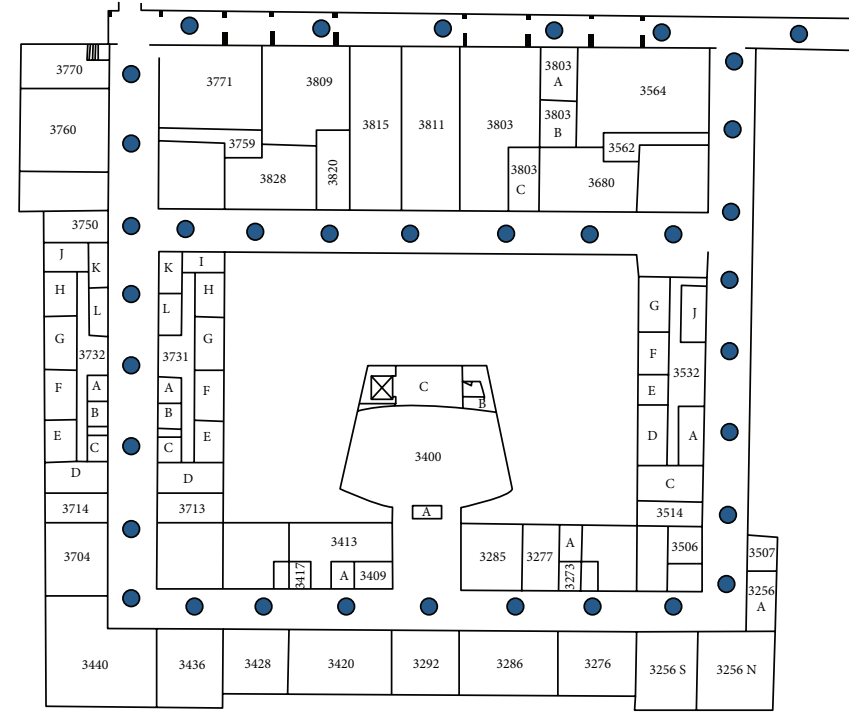

(b) 3rd floor of UCLA engineering building

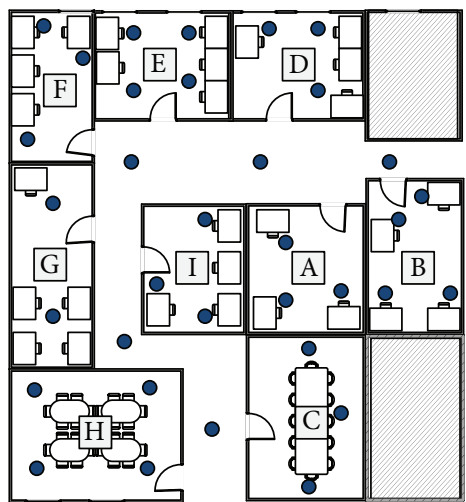

(d) UCLA NRL laboratory

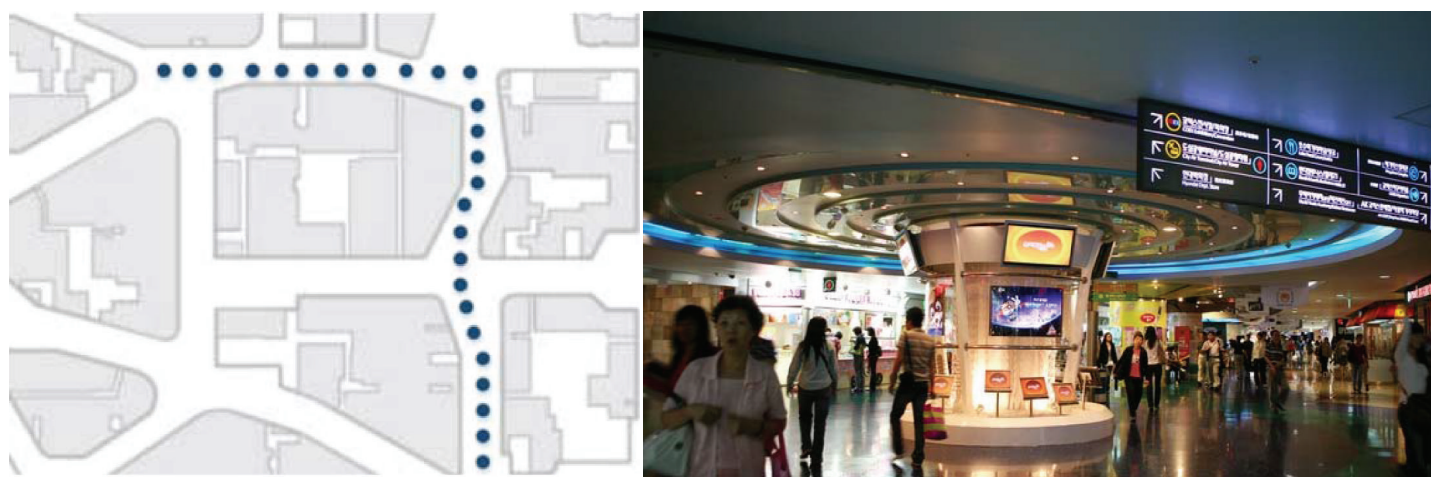

(e) Indoor Coex shopping mall in Seoul

FIGURE 2: Floorplans for evaluation.

other detected APs nearby (AP3-AP6 dBm) that show lower signal strength by more than $10 \mathrm{dBm}$ (i.e., the $\delta$ value).

From now on, we describe details of Freeloc localization algorithm in Algorithm 1. In the aforementioned scenario, users $B$ and $C$ request their positions to a localization server with own fingerprint values in Table 2, and then the fingerprint values are compared with the stored fingerprint map that is created by users A and D. First, AP4 as the strongest AP of user B is searched in Room 101 map. AP4 in the map has other APs, AP5, and AP6 that are weaker by the delta. Since AP5 and AP6 are commonly found in both the user B's fingerprint and the location map, Room 101 earns two points. Like this, our algorithm finally acquires 8 points for Room 101 but only 1 for the 102. For the user C, the scores are 9 and 


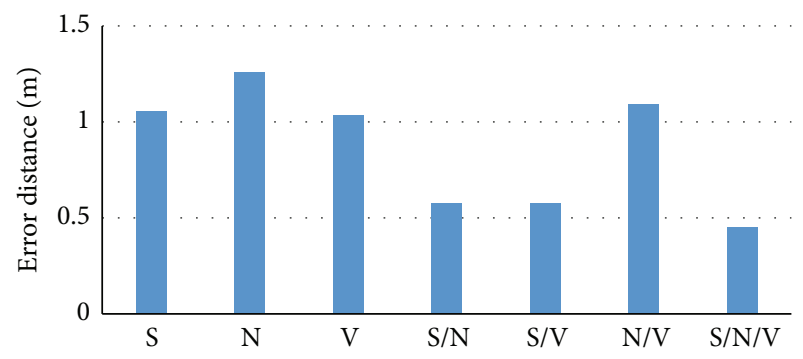

(a) Desire HD

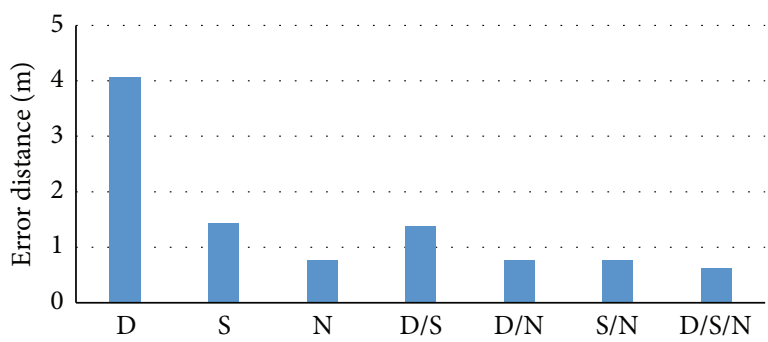

(c) Vega Iron 2

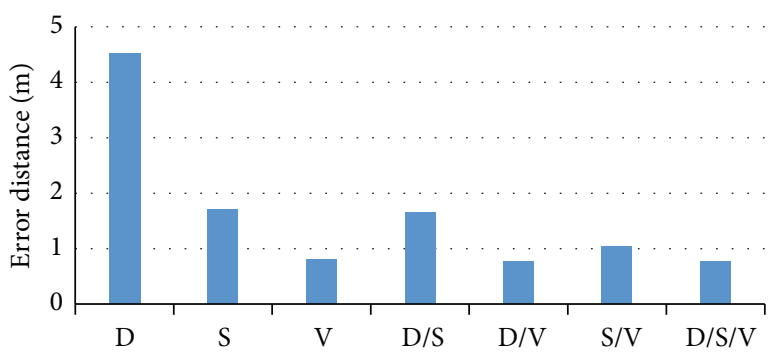

(b) Galaxy Note 4

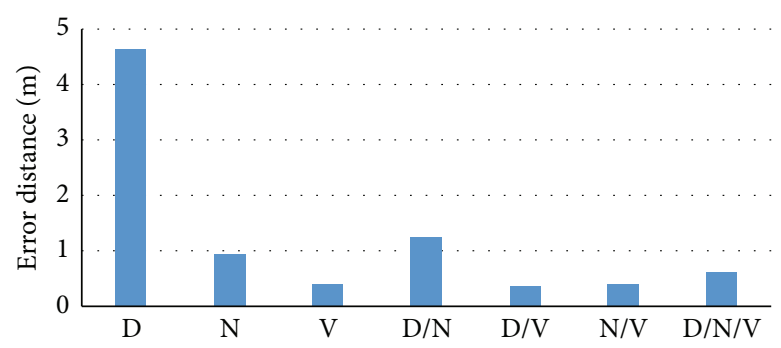

(d) Galaxy S3

FIGURE 3: Impact of device heterogeneity experiment at 5th floor of Gachon IT building.

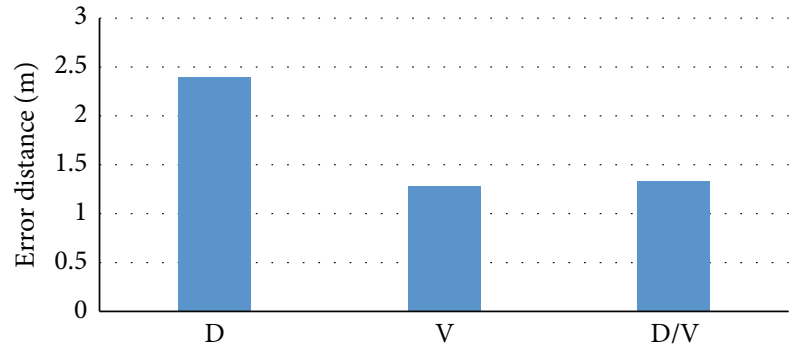

(a) Galaxy S3

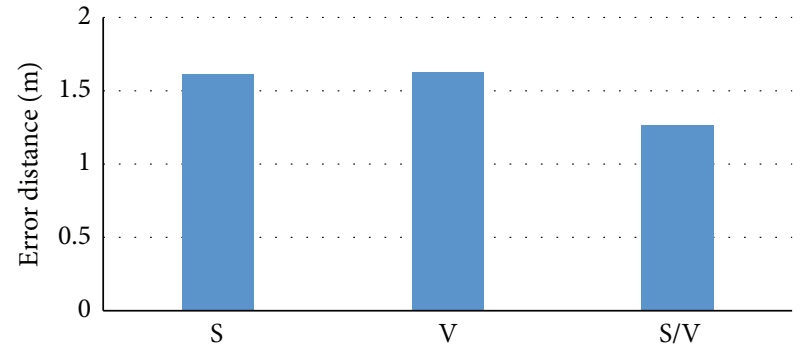

(b) Desire HD

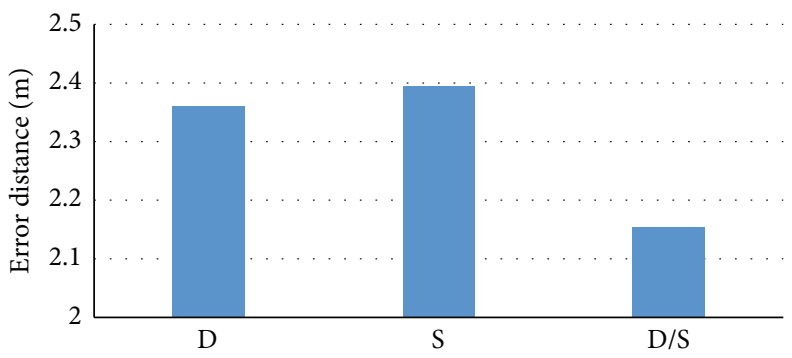

(c) Vega Iron 2

FIGURE 4: Distance errors of localization at a resident house.

2 for Room 101 and 102, respectively. Therefore we conclude that both users B and C are in Room 101.

In order to achieve fast retrieval (line 4 in Algorithm 1), we utilize an "importance flag," a one-bit flag in fingerprint data, Table 2. The flag is set if the $\mathrm{AP}_{i}$ ranks high with strong RSS. When the localization server receives such location request, it selects fingerprint data with flagged $\mathrm{AP}_{i}$ in the map which matches the flagged $\mathrm{AP}_{i}$ in the request data.

For example, in the same aforementioned scenario, the flagged $\mathrm{AP}_{i} \mathrm{~s}$ are $\{\mathrm{AP} 1$ and AP2 $\}$ for Room 101 and AP6 and AP5 for Room 102 if we set the criterion for the flag as the top two APs. The flagged $\mathrm{AP}_{i}$ s are marked with asterisk in Table 2. When users $B$ and $C$ request their positions, at least one flagged $\mathrm{AP}_{i}^{*}$ in their fingerprint data is found in the fingerprint data of Room 101 (i.e., AP1 for user B and AP2 for user C) while no matching flagged AP is found in the fingerprint data of Room 102. Therefore, only Room 101 is considered for the possible location in this example. However, we can be bothered from high rank $\mathrm{AP}_{i}^{*} s$ if adjacent locations have high probability of receiving strong RSS from the same APs. If no matches are found, we regard the position where the measurement is taken as an unlabeled location. 
TABLE 3: Measured fingerprint data for localization.

(a)

\begin{tabular}{lc}
\hline \multicolumn{2}{c}{ User B } \\
$\mathrm{AP}_{i}$ & $\mathrm{AP}_{j, \delta(i)}$ \\
\hline $\mathrm{AP}^{*}$ & $\{\mathrm{AP} 1, \mathrm{AP} 2, \mathrm{AP} 3, \mathrm{AP} 5, \mathrm{AP} 6\}$ \\
$\mathrm{AP1}^{*}$ & $\{\mathrm{AP} 5, \mathrm{AP} 6\}$ \\
$\mathrm{AP} 2$ & $\{\mathrm{AP} 5, \mathrm{AP} 6\}$ \\
$\mathrm{AP} 3$ & $\{\mathrm{AP} 5, \mathrm{AP} 6\}$ \\
$\mathrm{AP} 6$ & \{\} \\
$\mathrm{AP5}$ & \{\} \\
\hline
\end{tabular}

(b)

\begin{tabular}{lc}
\hline & User $\mathrm{C}$ \\
$\mathrm{AP}_{i}$ & $\mathrm{AP}_{j, \delta(i)}$ \\
\hline $\mathrm{AP}^{*}$ & $\{\mathrm{AP} 1, \mathrm{AP} 4, \mathrm{AP} 5, \mathrm{AP} 6\}$ \\
$\mathrm{AP}^{*}$ & $\{\mathrm{AP} 1, \mathrm{AP} 4, \mathrm{AP} 5, \mathrm{AP} 6\}$ \\
$\mathrm{AP} 1$ & $\{\mathrm{AP} 5, \mathrm{AP} 6\}$ \\
$\mathrm{AP} 4$ & $\{\mathrm{AP} 5, \mathrm{AP} 6\}$ \\
$\mathrm{AP5}$ & \{\} \\
$\mathrm{AP6}$ & \{\} \\
\hline
\end{tabular}

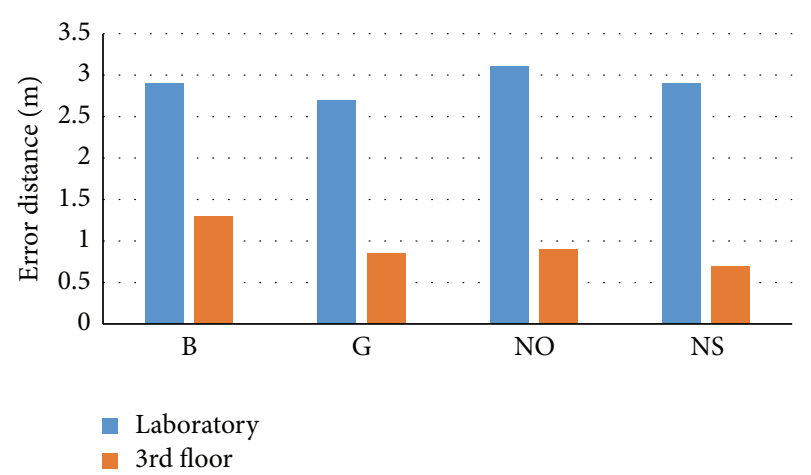

FIGURE 5: Localization accuracy comparison between laboratory and third floor in UCLA building [24].

4.2.2. Fingerprint Update. In CIL, mobile users as surveyors upload continuously different fingerprints for the same location with their heterogeneous devices, which increases the number of RSS data sets for the same location label in the map database and time to compare all sets for localization.

Freeloc can provide a simple update procedure that builds a unique fingerprint without any calibration, which just merges multiple fingerprints into a single fingerprint. For example, the new fingerprint data will be established as in Table 4 if B uploads its RSS measurements with the same location label as shown in Table 3 and they merge with an existing Room 101 fingerprint in Table 2 created by user A. The proposed $\left\{\mathrm{AP}_{i}, L\left(\mathrm{AP}_{j, \delta(i)}\right)\right\}$ fingerprint data structure and the $\delta$ value increase the similarity among multiple fingerprint data although they are measured at slightly different locations with different devices.
TABLE 4: Merged fingerprint data for Room 101.

\begin{tabular}{lc}
\hline \multicolumn{2}{c}{ Room 101: user $\mathrm{A}+\mathrm{B}$} \\
$\mathrm{AP}_{i}$ & $\mathrm{AP}_{j, \delta(i)}$ \\
\hline $\mathrm{AP} 1$ & $\{\mathrm{AP} 3, \mathrm{AP} 4, \mathrm{AP} 5, \mathrm{AP} 6\}$ \\
$\mathrm{AP} 2$ & $\{\mathrm{AP} 3, \mathrm{AP} 4, \mathrm{AP} 5, \mathrm{AP} 6\}$ \\
AP3 & $\{\mathrm{AP} 5, \mathrm{AP} 6\}$ \\
AP4 & $\{\mathrm{AP} 1, \mathrm{AP} 2, \mathrm{AP} 3, \mathrm{AP} 5, \mathrm{AP} 6\}$ \\
AP5 & \{\} \\
AP6 & \{\} \\
\hline
\end{tabular}

TABle 5: Devices used for data collection.

\begin{tabular}{lccc}
\hline Device & MFR & Wi-Fi chipset & Android \\
\hline Bionic (B) & Motorola & TI WL1285C & 2.3 .4 \\
Galaxy S2 (G) & Samsung & BCM4330 & 2.3 .4 \\
Galaxy S3 (S) & Samsung & M2322007 & 4.3 \\
Galaxy Note 4 (N) & Samsung & BCM4358 & 5.1 \\
Desire HD (D) & HTC & BCM4329 & 4.1 .2 \\
Vega Iron 2 (V) & Pantech & & 4.4 .2 \\
Nexus One (NO) & HTC & BCM4329EKUBG & 2.3 .3 \\
Nexus S (NS) & Samsung & BCM4329GKUBG & 2.3 .6 \\
\hline
\end{tabular}

\section{Performance Comparison at Different Environment}

From this section, we evaluate the Freeloc with 8 multivendor smart phones in Table 5 which have different Wi-Fi hardware chipsets and mobile OSs. For evaluation, we varied the number and type of devices which participate in the fingerprint map for each user device. The same device was never used for both generating fingerprinting map and localization.

5.1. Building Floor. First, 5th floor of the IT building at Gachon University and 3rd floor of the UCLA engineering building as office/school building environment where long corridors and surrounded meeting rooms and laboratories exist as shown in Figures 2(a) and 2(b). The width of the corridor is approximately $2 \mathrm{~m}$, and some APs are visible with line-of-sight. Their floor plan is similar to each other, but WiFi AP density is different; Gachon IT building has more than 300 detected APs while the UCLA building captures about 50 APs. We measured RSSs at 36 different positions at the third floor of the engineering buildings in UCLA campus and 35 positions at the IT building of Gachon University. Small dots in figure indicate the locations where RSS measurements were taken. The data collected at these points were used for experiments. The adjacent points are approximately $6 \mathrm{~m}$ apart.

In the experiment, Freeloc localization algorithm is demonstrated for feasibility of CIL with heterogeneous multiple devices at corridor based office buildings. Localization results from the heterogeneous devices at the Gachon University are shown in Figure 3. First three bars are the cases when only one device was used for the fingerprinting. The next three bars are the cases when the fingerprint maps were generated from two different devices and these fingerprint 

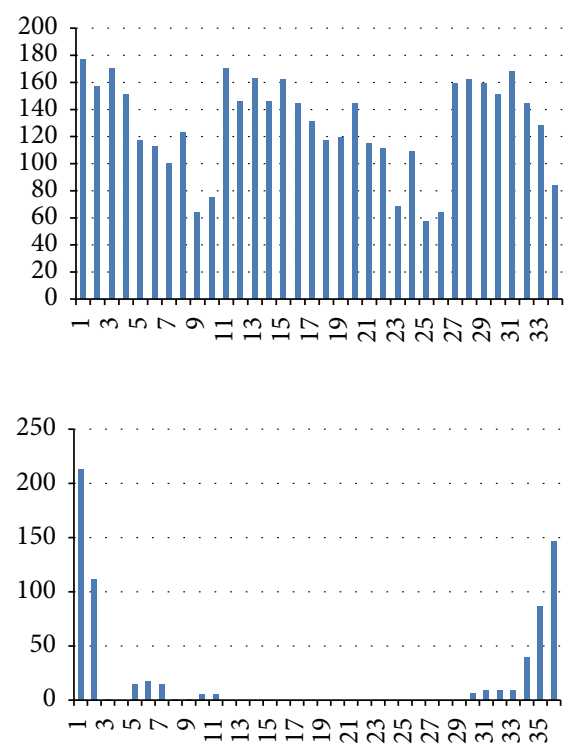

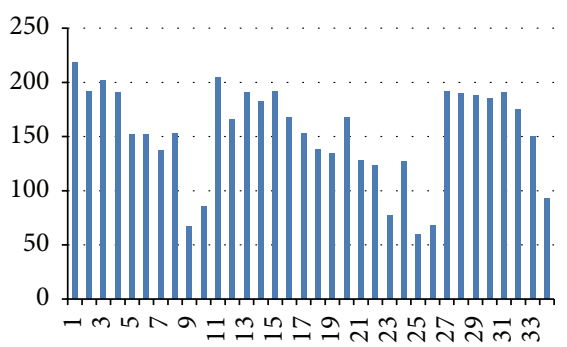

(a) Score at laboratory at positions 1-3

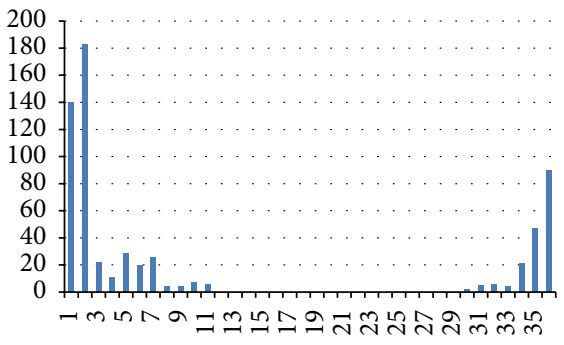

(b) Score at 3rd floor at positions 1-3
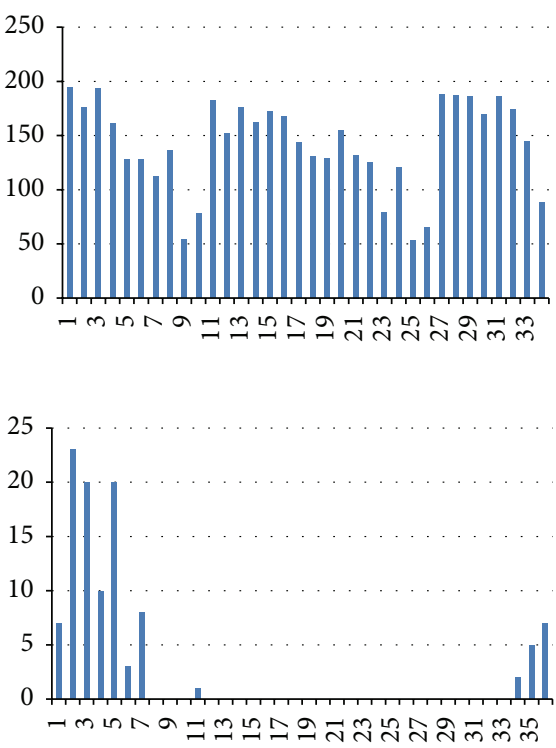

FIGURE 6: Localization scores at measurement locations of UCLA laboratory and 3rd floor.

maps were merged to form one fingerprint map. The last bar denotes the case when fingerprint maps have been generated from three different devices and merged.

Figure 3 shows that the accuracy did not degrade mostly when more than one fingerprint data from different devices are merged. Particularly, HTC Desire HD (D) results in higher distance error to any fingerprint created by other phones. The distance error has been observed exceeding $4 \mathrm{~m}$ with the Desire HD. But the distance error decreases with additional fingerprints combined by other heterogeneous devices to less than $1 \mathrm{~m}$. Herein we can conjecture that more additional measurements from other heterogeneous devices contribute to reduce the distance error. Furthermore, the distance error will decrease considerably as the homogeneity of devices increase in the crowdsourced system.

Experiment results in Gachon and UCLA buildings [24] show that Freeloc can provide consistent performance among heterogeneous devices. According to our observation in Figure 5 [24], error distance in Gachon IT building is less than UCLA engineering building, about 1 versus $3 \mathrm{~m}$, which implies that the number of APs and degree of their distribution can affect localization performance in the Freeloc.

5.2. Residential Area. Next localization experiment for small residential area had been conducted at UCLA laboratory and a residential house, which have many different types of furniture and only a few residential people stay. However, the UCLA laboratory is partitioned by thin plywood for rooms and the partitions do not reach to ceiling while the residential house has rooms separated by thick concrete walls, for example, a living room, a kitchen, and a restroom. In the residential house and UCLA laboratory, 29 and 34 positions are measured, respectively. Each of the measurement points is approximately $1.5 \mathrm{~m}$ apart.
Figure 4 depicts distance errors of a resident house with three heterogeneous devices (D, S, and V). Cross-device error exceeds $1.5 \mathrm{~m}$ that is a distance between measurement positions in case of localization with a single fingerprint. However, the distance error becomes less than the $1.5 \mathrm{~m}$ with two fingerprints given by heterogeneous devices. In all cases of the figure, the accuracy did not degrade when more than one fingerprint data from different devices are merged. This reaffirms that the Freeloc can be a feasible solution for the CIL.

The localization performance at the resident house shows better than UCLA laboratory in Figure 5 even though their sizes are similar. We can conclude that the residential house can obtain unique and stable fingerprints rather than the UCLA laboratory since the rooms of the resident house are separated by the concrete walls and channel variation caused by residential people is smaller (e.g., more than 10 students in the UCLA laboratory and 2 residents in the house).

Figure 5 shows that the average distance error at the UCLA laboratory compared to the third floor of UCLA engineering building. The distance error at the UCLA laboratory is higher the third floor where the measurement point interval is longer than the laboratory (1.5 versus $6 \mathrm{~m}$ ).

The reason for this observation of degraded accuracy in the laboratory can be difficulty to have a unique fingerprint compared to the third floor that has more unique fingerprints formed with thick concrete walls of the building due to path loss while the laboratory is made of thin plywood material for rooms inside.

Figure 6 shows score distribution derived from Algorithm 1 at the measurement position from 1 to 3 , where localization error occurs at positions 2 and 3 of the laboratory and position 3 of the third floor. As can be seen in the figure, the scores of the laboratory are very similar to 


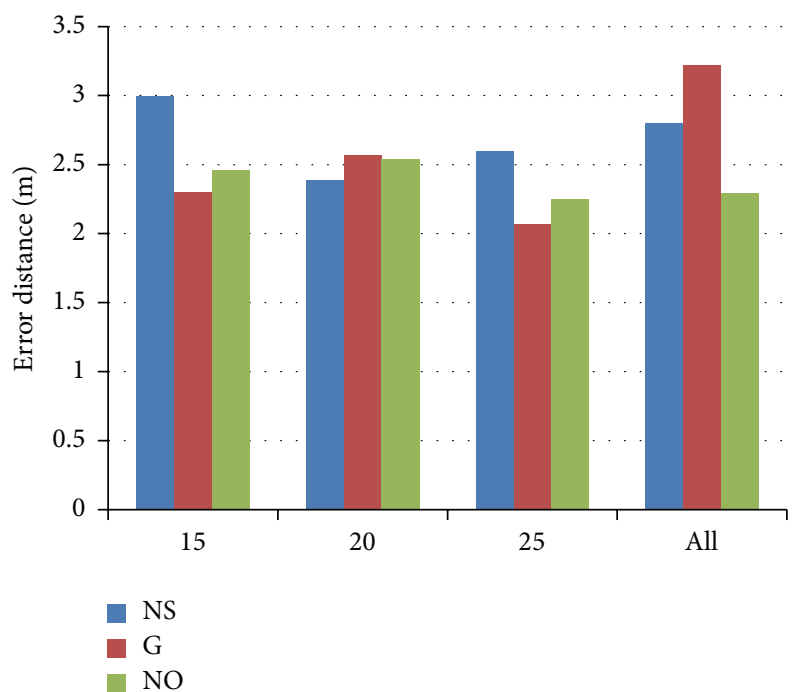

(a) 34 positions

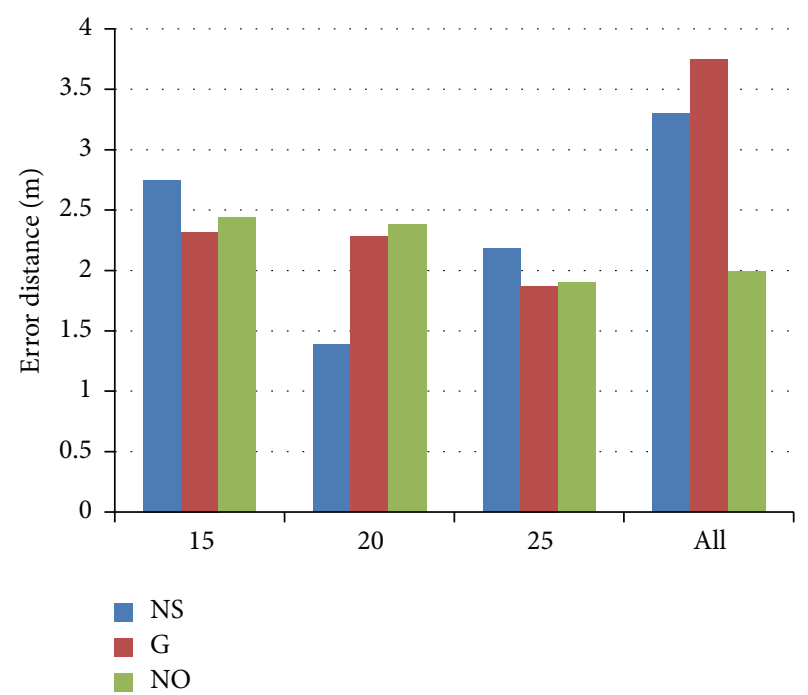

(b) 17 positions

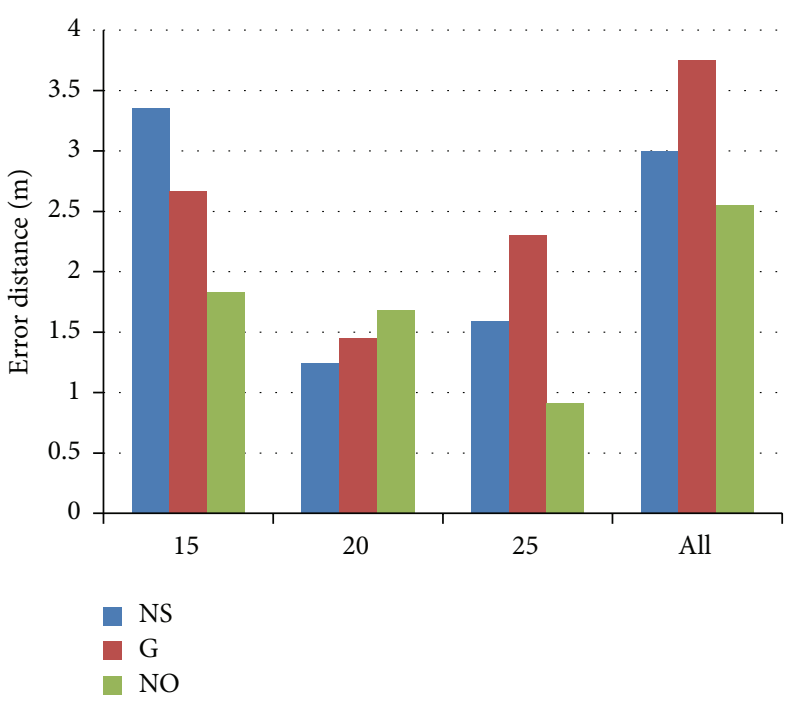

(c) 8 positions

Figure 7: Analysis of localization performance at small region. Distance errors of Bionic user device with a reduced number of APs and measurement positions.

each other among measurement locations compared to the third floor, which leads to wrong prediction with higher probability than the third-floor case.

According to our analysis on the laboratory fingerprints, we observed that the similarity of the fingerprints is caused by similar order of the fingerprints. That is to say, fingerprints in the laboratory have almost the same sequence of AP BSSIDs due to plywood walls except several of the strongest APs that should be highlighted. To see impact of measurement distance and number of APs for a fingerprint, twofold is revisited as inputs for fingerprints as shown in Figure 7.

Since the short measurement distance at the small area like a laboratory may increase localization error probability compared to the corridor based office buildings, we experimented our localization algorithm with smaller number of measurement spots. With many APs in similar order, a long list of measured APs increases the score value and make the fingerprint undistinguishable. Thus, we tested with a reduced number of APs, for example, the strongest 15, 20, and 25 APs from more than 40 captured APs in the laboratory.

In Figure 7(a), 20 APs are most preferable for the fingerprint of the laboratory. The distance error decreases to 2.4 and $2.5 \mathrm{~m}$ from 2.8 and $3.2 \mathrm{~m}$ in NexusS (NS) and Galaxy $(\mathrm{G})$, respectively. This error decreases more if the number of measured sites decreases by a half. The distance error of the NexusS and Galaxy decreases to 1.39 and $2.28 \mathrm{~m}$ as shown in Figure 7(b). The distance error decreases more with smaller measurement sites in Figure 7(c). Table 6 shows average distance error with a number of APs for the fingerprints. Without resizing a list of captured APs, the average distance error was not decreased only by reducing the number of measurement sites (little bit increase in case of $A l l$ ). On 


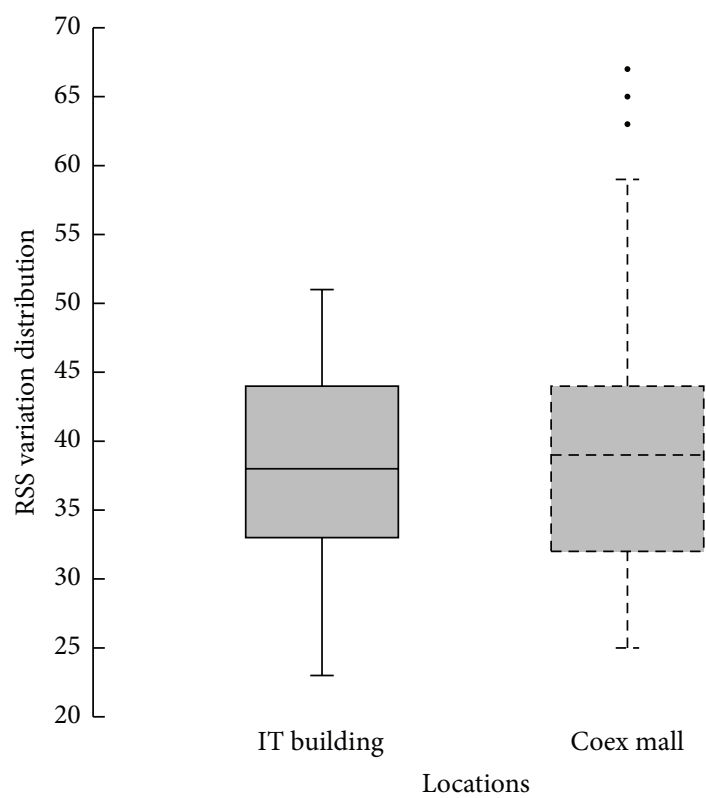

FIgURE 8: RSS variation at Gachon IT building and Coex. - represents the exceptional cases that are out of $95 \%$ confidence interval.

TABLE 6: Average distance error with a reduced number of APs.

\begin{tabular}{lccc}
\hline APs & 34 sites & 17 sites & 8 sites \\
\hline 15 & 2.58 & 2.5 & 2.61 \\
20 & 2.5 & 2.01 & 1.45 \\
25 & 2.31 & 1.98 & 1.6 \\
All & 2.77 & 3.01 & 3.08 \\
\hline
\end{tabular}

contrary, the average distance error decreases from 2.5 to $1.45 \mathrm{~m}$ with $20 \mathrm{APs}$ if the measurement sites are reduced from 34 to 8 sites. Such reduction of AP sequence can also decrease location estimation time that is proportional to the number of fingerprint data to be compared, in addition to give a highlight to the strong APs for a fingerprint.

Localization with fine granularity is still challenge especially in small open space where similar fingerprints are captured. Accordingly, measurement position distance or a number of APs used for fingerprints should be adjusted according to the localization environment, especially for the small area. However, we argue that Freeloc is still feasible for home localization as one of key technologies for Internet of Things (IoT).

5.3. Indoor Shopping Center. Wi-Fi channels at indoor environment are varying with moving objects, especially, people, which leads to variance in measured fingerprints. We can conjecture that the fingerprint abstraction based on (4) can deal with this problem. However, largely distributed RSSs can still mislead the localization with inconsistent fingerprints.

In order to demonstrate this problem, we experimented our localization method at the crowd indoor shopping mall, the Coex, one of the biggest indoor shopping centers in south Korea, which holds large convention rooms for exhibitions and conferences, many restaurants, and stores (Figure 2(e)).
The Wi-Fi fingerprint data were collected at 40 positions for different Wi-Fi channel environment and each position is about $6 \mathrm{~m}$ apart the same as in the school buildings. We measured RSSs from more than 200 APs installed by Coex, stores, and Internet service providers.

We used four smart phones, Desire HD (D), Vega Iron (V), Galaxy S3 (S), and Note $4(\mathrm{~N})$ and measured RSSs with each phone at different time to experience different channels. To investigate degree of fading effect from pedestrians, RSS variation of two different places, Gachon IT building and Coex mall, is compared in Figure 8. The RSS variation in the Coex mall is higher than the IT building probably due to more pedestrians; RSS difference is almost about $10 \mathrm{~dB}$ in some of APs. However, the RSS distribution of strong APs is very similar in both locations.

Figure 9 shows error distance of pairwise experiments of those heterogeneous devices at the Coex mall. The distance error is bigger than the Gachon IT and UCLA building due to varying channel status even though more APs were captured for fingerprints. Also, measurement by Desire HD leads to increase distance error. However, the error distance decreases with fingerprints gathered by other three heterogeneous devices up to less than measurement distance, $6 \mathrm{~m}$. According to this experiment results, localization performance for CIL is affected more by device characteristics, that is, heterogeneity rather than fading channel status because RSS value abstraction based on multiple measurement samples (e.g., more or less 10 samples) reduces the fading effect. Thus, Freeloc can be robust even in the crowd indoor environment that suffers from severe channel fading due to pedestrians.

\section{Performance Comparison of Localization Algorithms}

First, localization performance is compared when using a Bionic device and three fingerprints built by others. Figure 10 shows CDF of error distance at the laboratory for each different fingerprint. Error distance is slightly different from the fingerprints but comparable except $k$-NN. Maximum error distance is however very different from the localization techniques. Tanimoto approach shows biggest error, about 12 meters, among the four localization schemes since similarity between RSS fingerprints is undistinguishable to each other at the narrow laboratory environment. Figure 11 shows CDF of error distance at the third floor. $k$-NN and Tanimoto approaches show considerable error compared to the $N$-gram and Freeloc. $N$-gram and Freeloc show only about 5-meter error at more than $80 \%$ fingerprints error distance while $k$ $\mathrm{NN}$ and Tanimoto have more than 30-meter distance error. Accordingly, algorithms using relative information for the fingerprint outperform ones using the absolute vale of the RSS, especially for third-floor environment. Comparing the Freeloc and $N$-gram, the Freeloc shows better accuracy since Freeloc can detect unique BSSID subsequence effectively even under varying radio condition.

Figure 12 shows total average error distance of four localization schemes using 4 user devices used in above experiment at the laboratory and third floor. "Single" indicates an average distance error of single pairwise evaluations. 


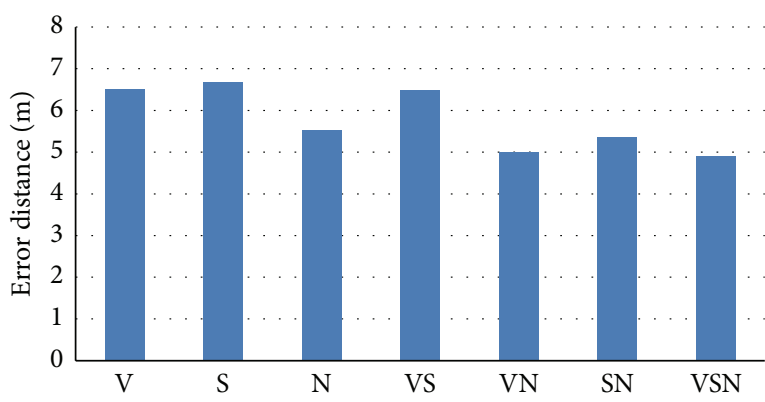

(a) Desire HD

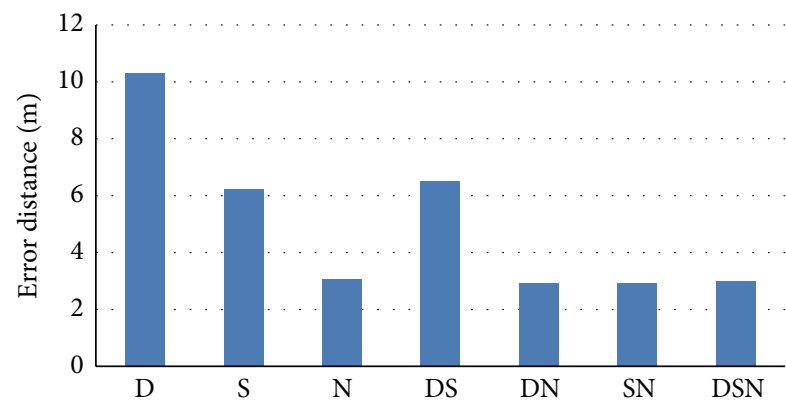

(c) Vega Iron 2

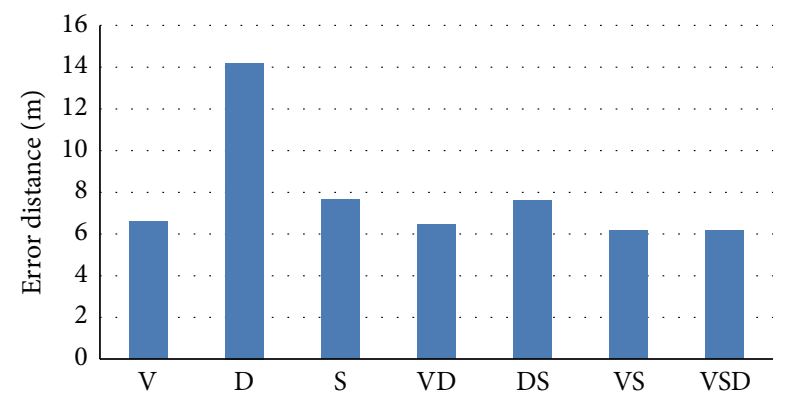

(b) Galaxy Note 4

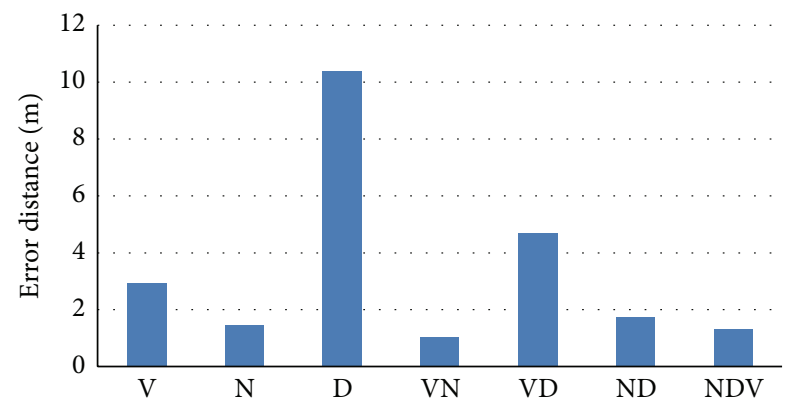

(d) Galaxy S3

FIgURE 9: Localization error at a crowd indoor place with many pedestrians.

At the laboratory, most algorithms show competitive results that error distance is below 5 meters. However, signal strength based localization techniques result in serious outcomes at the third floor. At the laboratory, RSSs of many fingerprints can help in elaborating localization accuracy but can mislead in wide area like third floor. Considering typical room size, it could be challenge in localization performance. Freeloc achieves competitive localization performance in both areas. Average error distance of localization based on heterogeneous multiple devices (i.e., 2 or 3 devices) indicates bars with the label "multiple" in Figure 12. When using multiple heterogeneous devices for building a radio map, $N$-gram and Freeloc that use relative information obtain notable gain compared to the single device surveying. On the contrary, distance error of $k$-NN and Tanimoto gets even worse than single device case. In $N$-gram and Freeloc, merged fingerprint data do not affect localization results because they only concern relative information of them. As some omitted fingerprints from a single device are filled by others appropriately, the accuracy is improved. However, in $k$-NN and Tanimoto, the same fingerprint is not merged properly and newly added values from other devices can mislead outcomes because heterogeneous surveying devices without calibration can get different RSS values for the same location. Error ratio that is a success rate of localization against trials at all measurement points is shown in Figure 12(c). Error ratio at the laboratory is higher than at 3rd floor because distance between measurement points is too small to detect exact location.

Figures 13 and 14 illustrate distribution of error distance at the laboratory and third floor, respectively. Error distribution indicates stability of the localization algorithms. Although $k$ NN shows lower error ratio at the laboratory in Figure 12(c), distance error is widely spread (max error distance is almost 11 meters) compared to the Freeloc. At the third floor, distance error of the Freeloc is also distributed within very short range than others including the $N$-gram of which error ratio is similar in Figure 12(c). Consequently, Freeloc can estimate a location with relatively low error variance rather than other localization schemes. In case of using multiple devices for fingerprint surveying, improvement compared to the single device case is not very notable. The heterogeneous device made fingerprints contribute to reducing error ratio in Figure 12(c), but not to reducing distribution of error distance much.

\section{Performance with Varying Delta Value}

In this section, we describe how to derive the delta value for Freeloc fingerprints, which was not handled in our previous work [24]. Practically, two delta value acquisition methods can be considered for Freeloc implementation; one is an instant derivation that occurs occasionally with several stored fingerprints and the other is an accumulated evaluation that occurs every time a new measurement is received.

7.1. Delta Derivation with Instant Fingerprints. To find out the optimal $\delta$ value for subsequent localization, the distance error of pairwise devices is calculated with $\delta$ values varied from 1 to 15 for all devices. From this, we can find the optimal empirical $\delta$ value which minimizes average distance error. Here, the error distance of the pairwise devices is calculated for the 


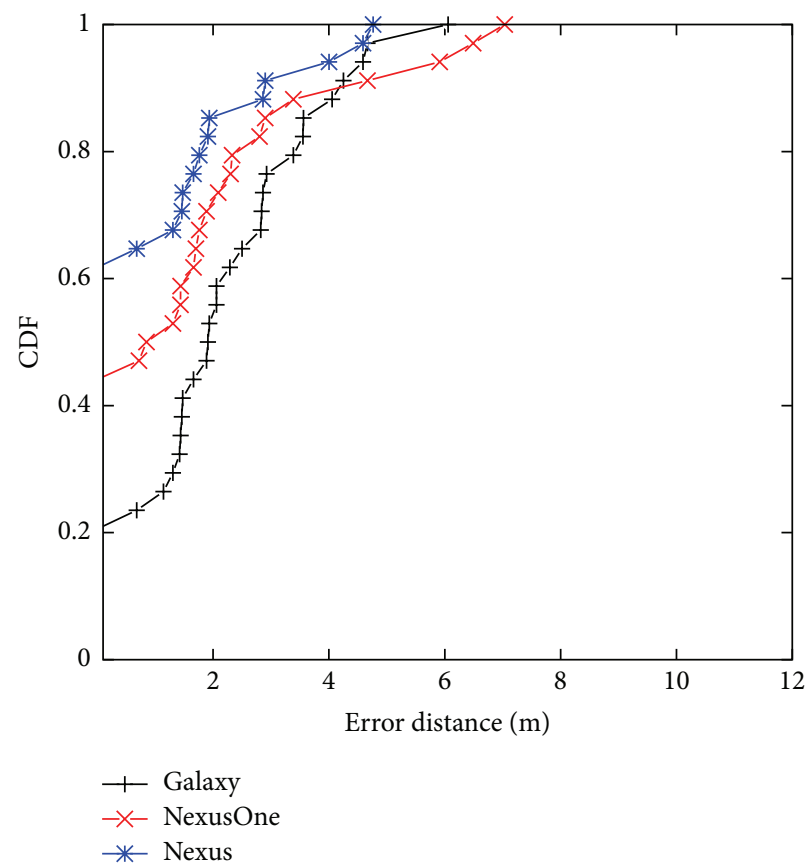

(a) $k$-NN

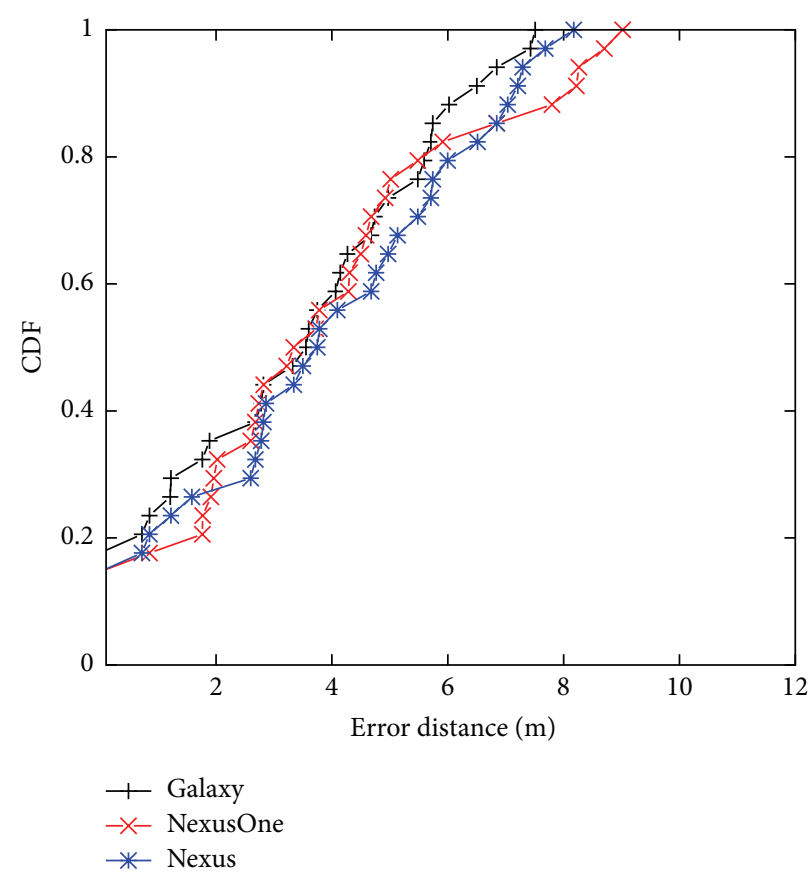

(c) $N$-gram

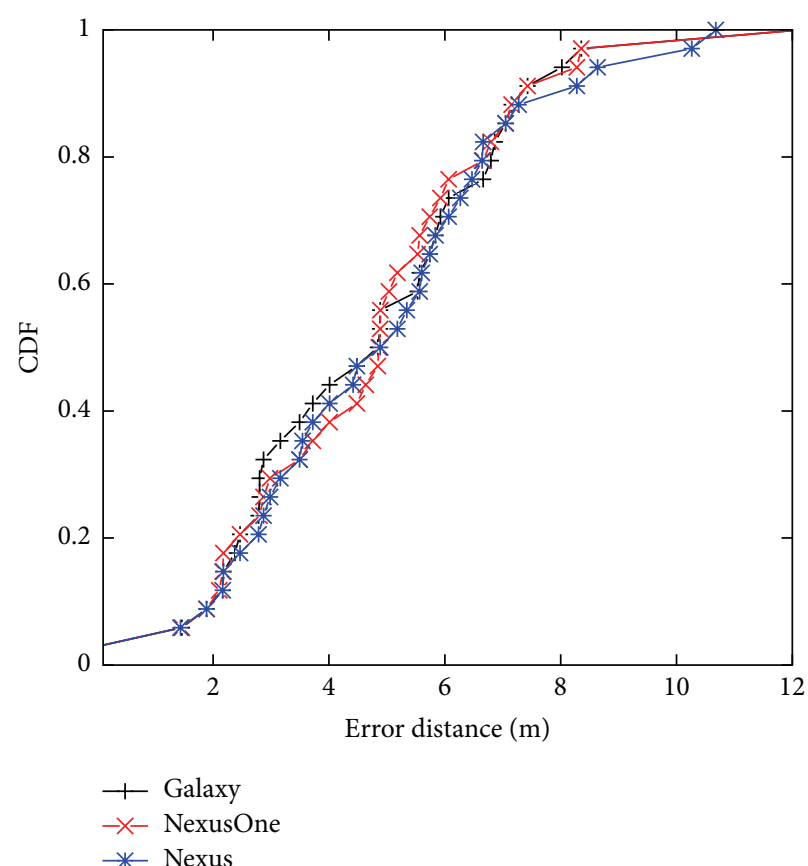

(b) Tanimoto similarity

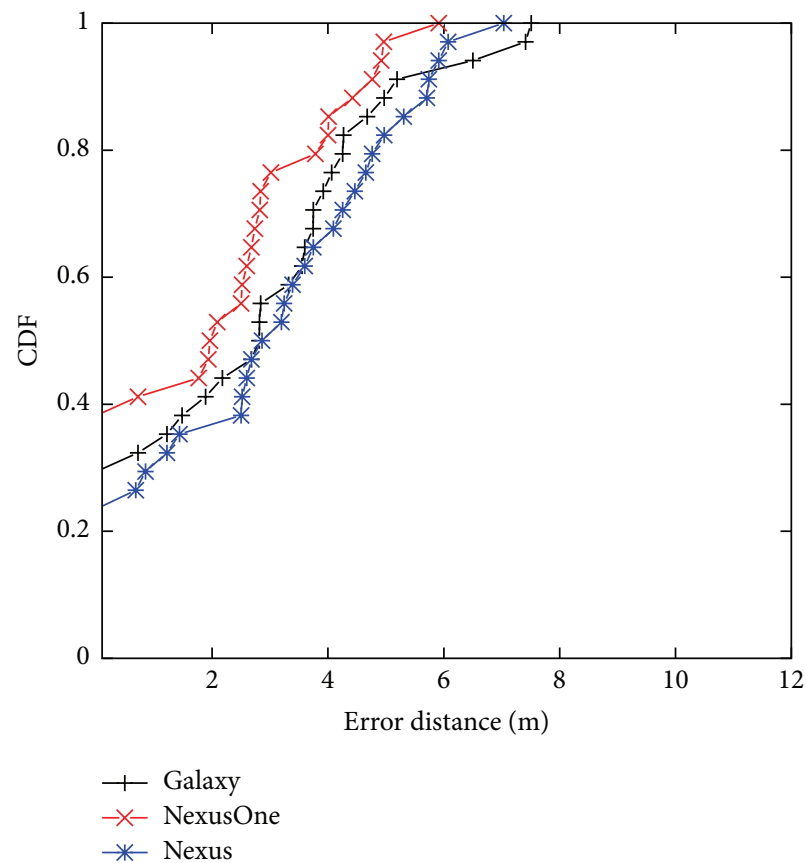

(d) Freeloc

FIgURE 10: Localization of a Bionic device at laboratory.

cases where the predicted location of the user was different from the real location. For instance, if fingerprints measured at point 1 in Figure 2(b) are regarded as fingerprints of point 2 by our localization algorithm, then error distance is an actual distance between those two measurement points.

For example, the instant average of each distance error from pairwise evaluation of four phones at the UCLA laboratory decreases until $\delta=12$ as shown in Figure 15(a).
Accordingly, optimal delta value can be set around $12 \mathrm{dBm}$ as noted in the average line in Figure 15. Practically, the localization server holds RSSs reported by several surveyors for the $\delta$ evaluation time and derives the optimal $\delta$ internally. This instant derivation can be performed periodically or occasionally to adapt to changed environment such as newly installed Wi-Fi APs and new type mobile devices. 


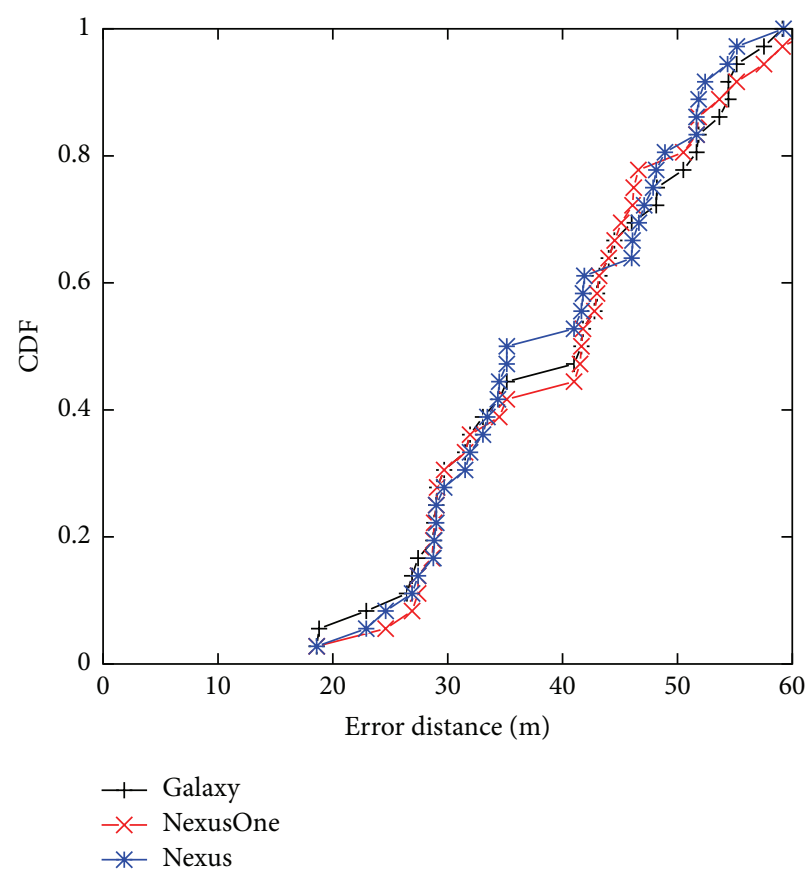

(a) $k$-NN

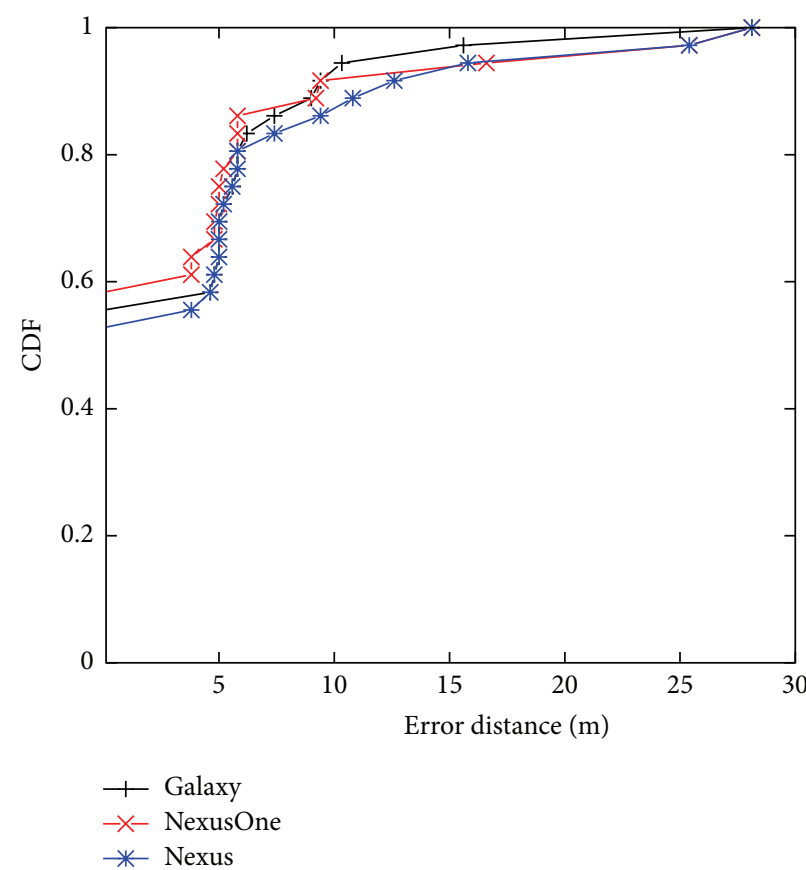

(c) $N$-gram

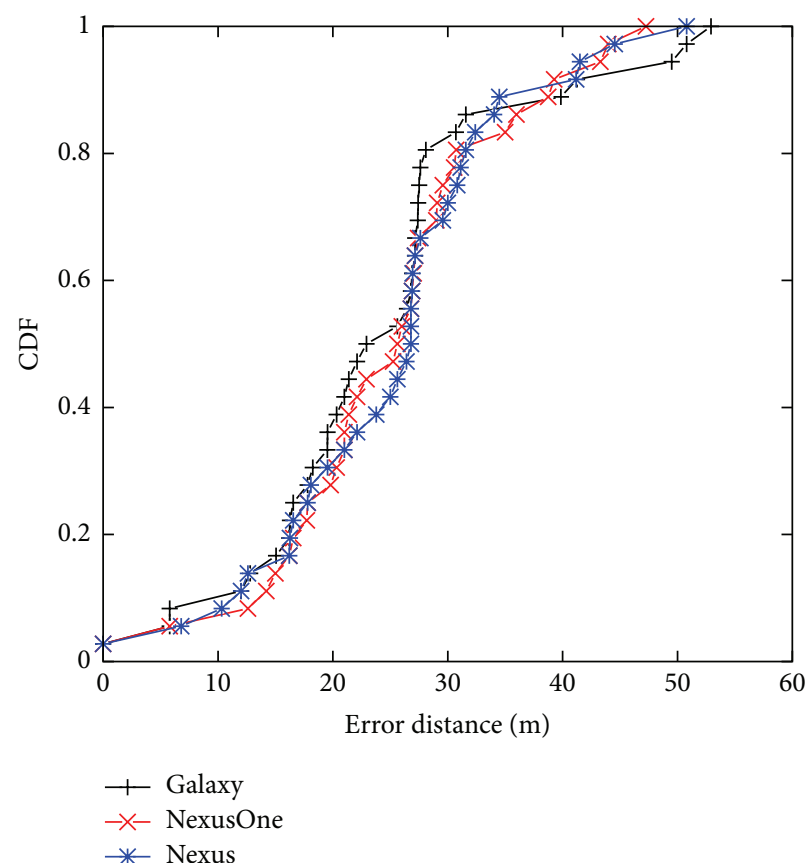

(b) Tanimoto similarity

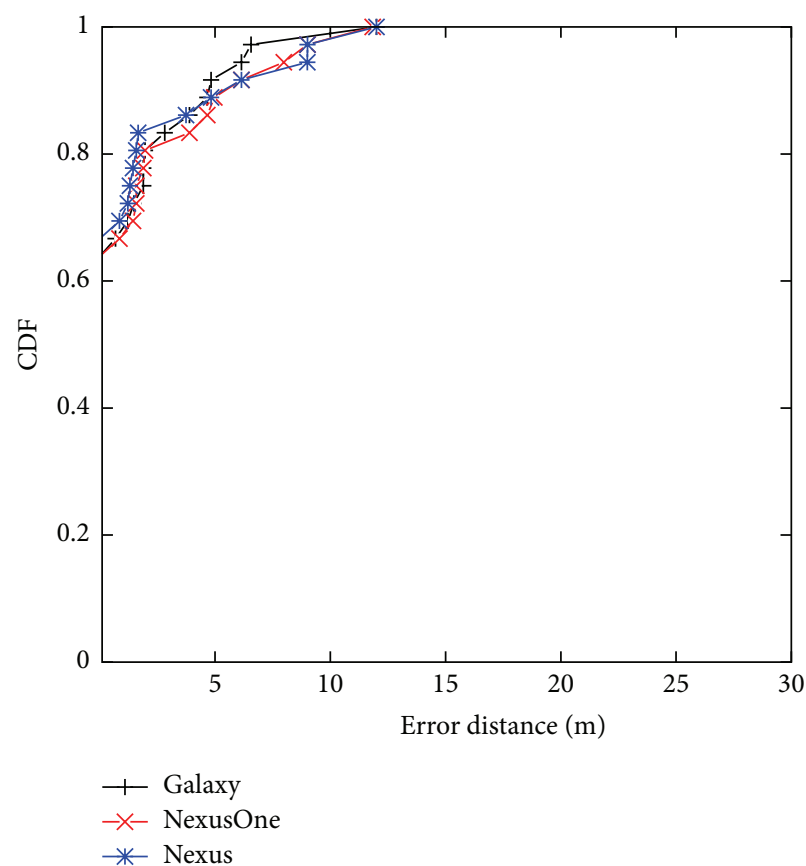

(d) Freeloc

FIgURE 11: Localization of a Bionic device at 3rd floor.

7.2. Delta Derivation with Accumulated Fingerprints. With accumulated fingerprints, the localization server derives a new optimal $\delta$ with a newly reported RSS from a phone based on the current fingerprint. For example, an initial fingerprint from the Bionic (B) is compared by the Galaxy (G) RSS that is reported later. With two, a first $\delta$ value is derived. After then, NexusS (NS) and NexusOne (NO) can update $\delta$, sequentially. Whenever the fingerprint is reported, it is used to derive $\delta$ combined to a single fingerprint per each location. Here, the localization server does not need to maintain raw RSS values of the reported fingerprints to derive $\delta$. The server only updates the current $\delta$ value based on fingerprints newly reported by surveyors. In Figure 15(b), for example, first the $\mathrm{B} / \mathrm{G}$ pair has an optimal $\delta$ in $14 \mathrm{dBm}$, and $\delta$ is updated to $12 \mathrm{dBm}$ by the NS. Finally, $\delta$ is set as $13 \mathrm{dBm}$ by the NO.

Figure 16 shows localization errors of the HTC Desire HD (D) with varying $\delta$ values at Gachon IT building. A merged fingerprint of two different devices does not show 


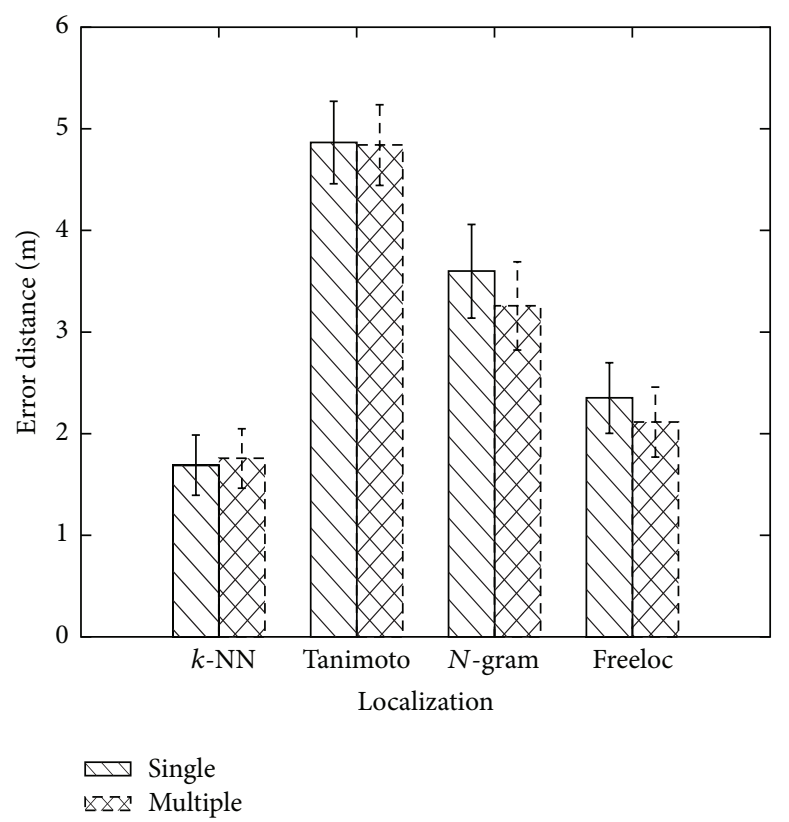

(a) Localization comparison at laboratory

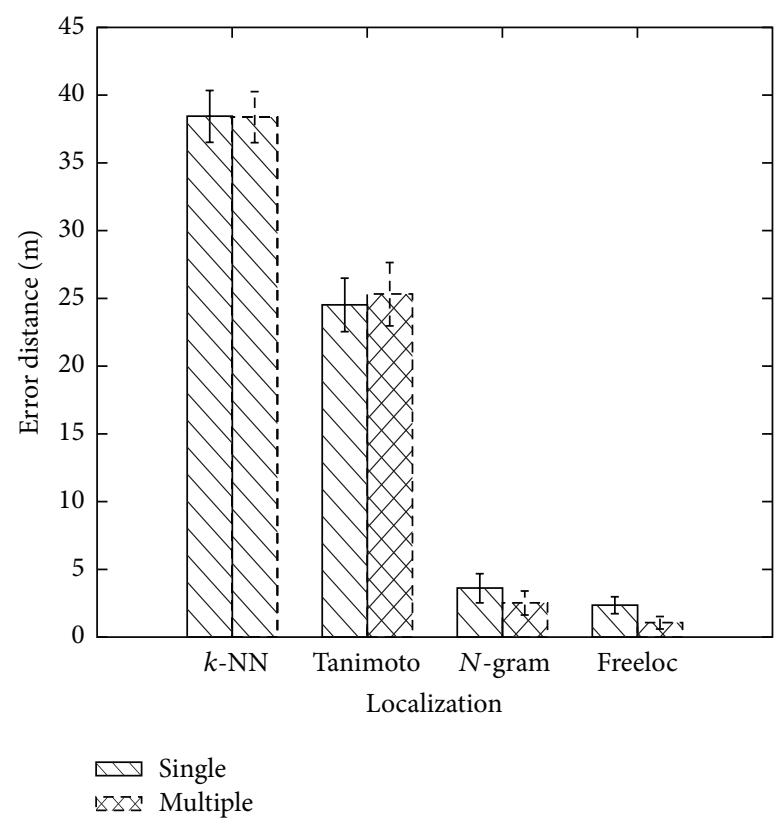

(b) Localization comparison at 3rd floor

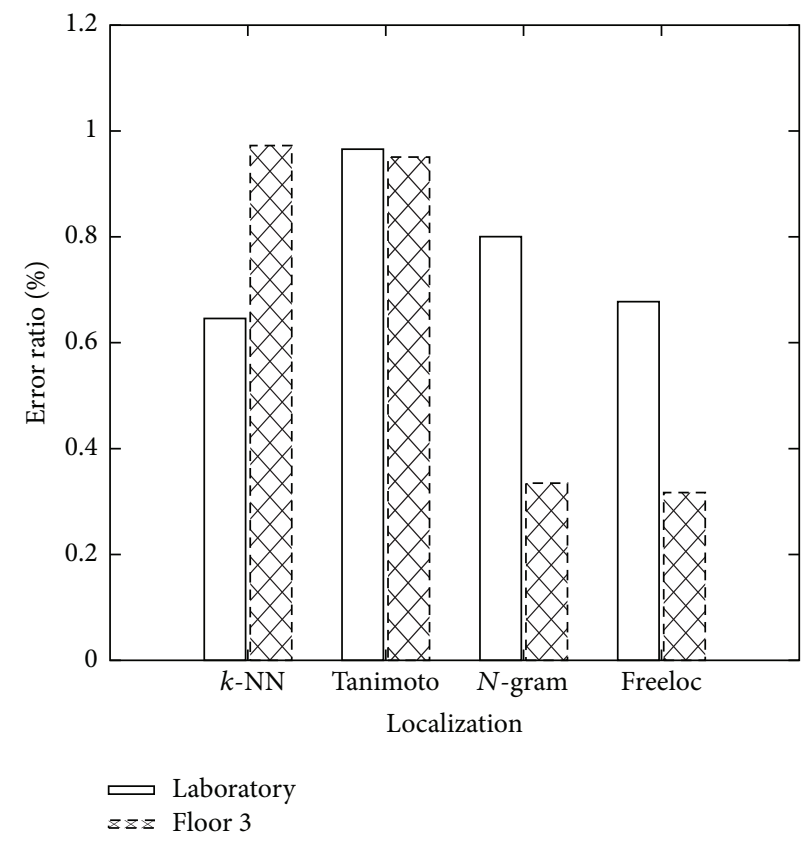

(c) Location detection error ratio

FIGURE 12: Comparison of different localization schemes.

better performance than a single device fingerprint in some of $\delta$ values. However, the most robust fingerprint has been formed from three different devices even though distance error is varying according to the $\delta$ value. This experiment justifies that our method can achieve impressive performance among heterogeneous devices in crowdsourced environment. We believe that error will decrease considerably as the homogeneity of devices increase in the crowdsourced system.

In this study, considering general performance in terms of total distance error, $10 \mathrm{dBm} \delta$ value is used for the UCLA engineering building and the Coex mall, and $2 \mathrm{dBm}$ is used for the Gachon IT building and the resident house in the rest of the evaluations.

\section{Conclusion}

FreeLoc is a novel, calibration-free indoor localization scheme that uses existing Wi-Fi infrastructure. The proposed radio map building and localization techniques are based on the overall relationship among RSS by APs. Our techniques provide robust localization accuracy in a crowdsourced environment in which device heterogeneity and multiple 


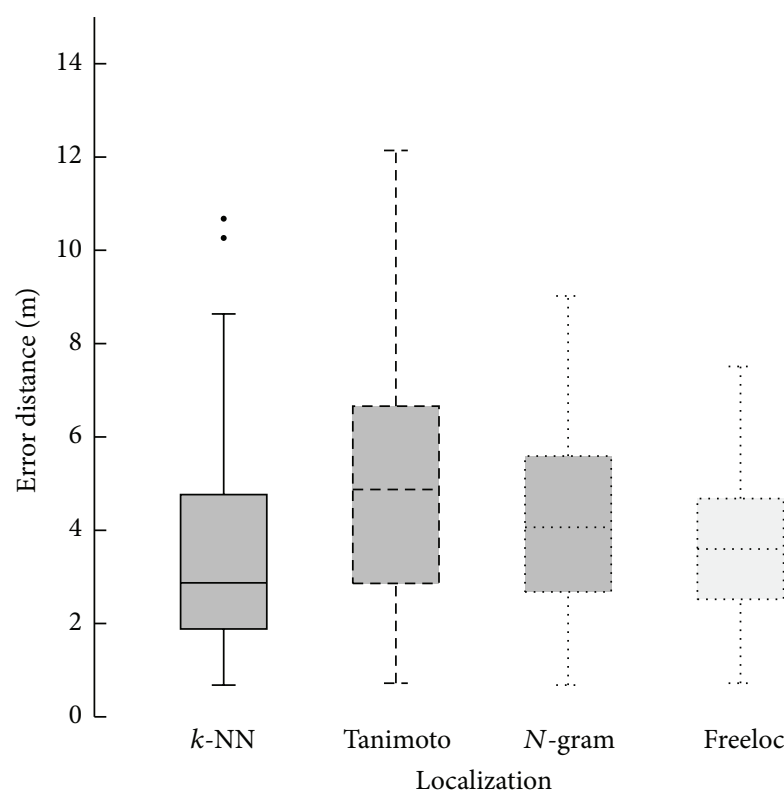

(a) Fingerprint of a single device

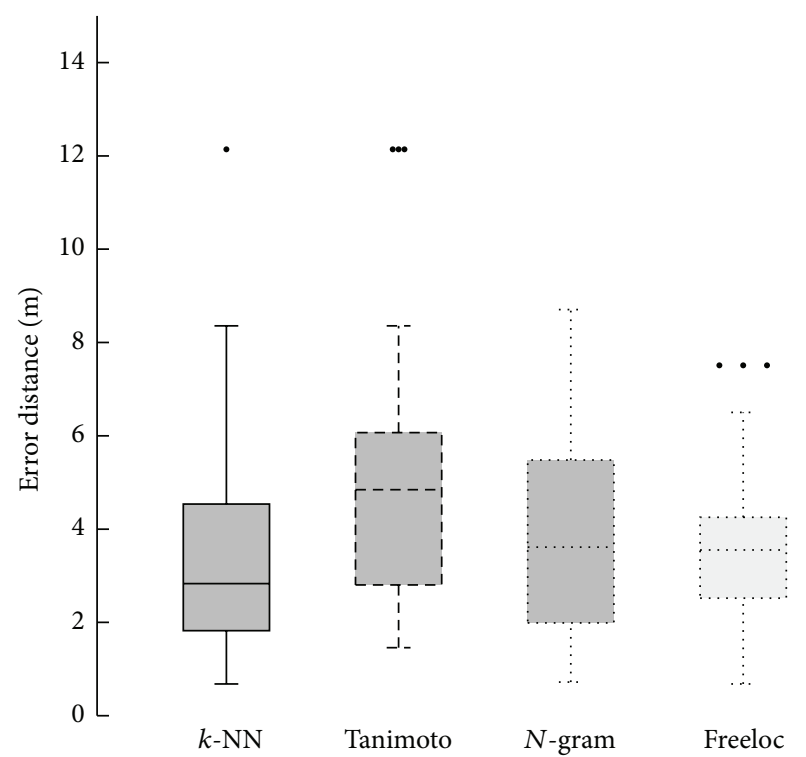

Localization

(b) Fingerprint of multiple devices

FIGURE 13: Error distributions at laboratory.

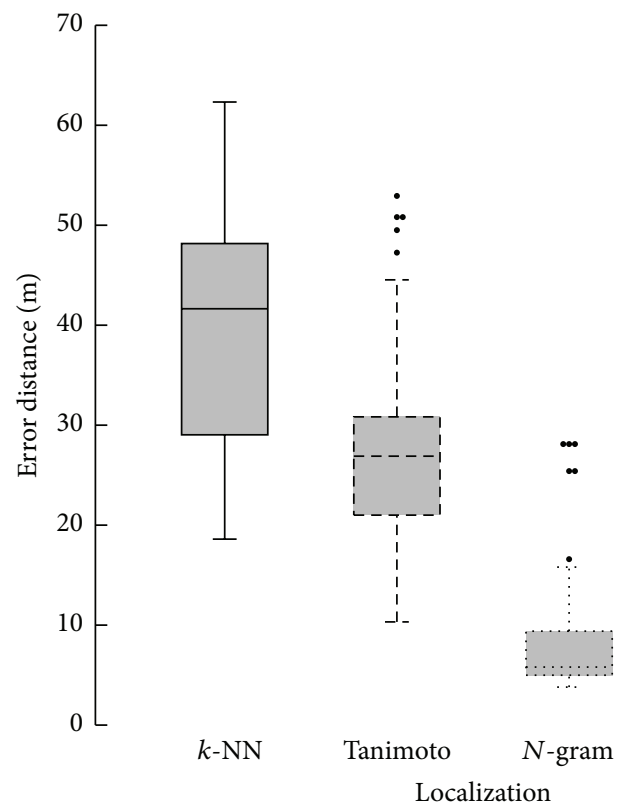

(a) Fingerprint of a single device

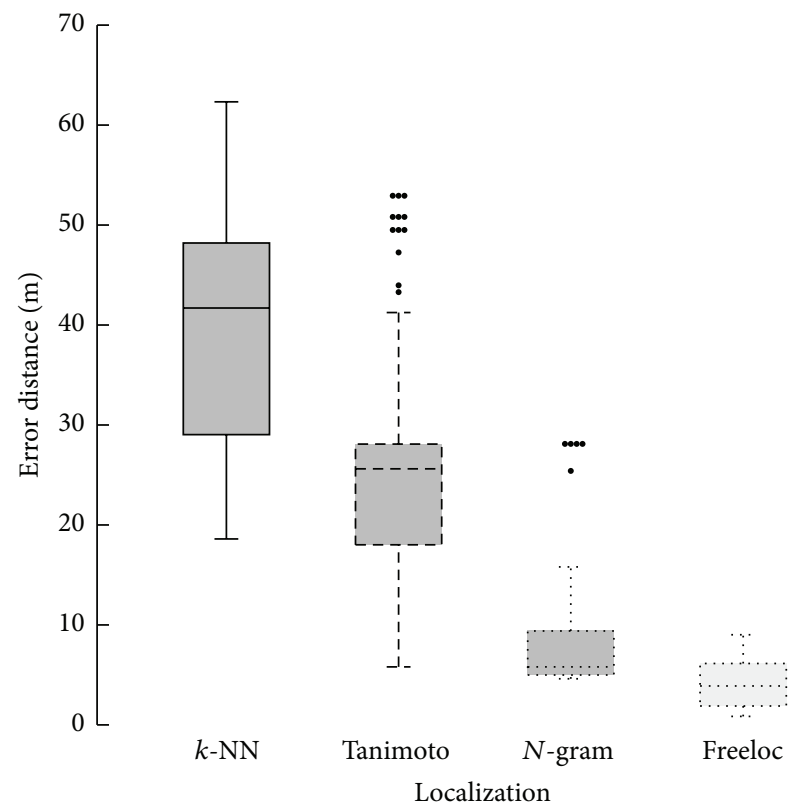

(b) Fingerprint of multiple devices

FIGURE 14: Error distributions at 3rd floor.

untrained surveyors mainly cause performance degradation. The main contribution of our work is a novel approach to fingerprint data management and a localization algorithm that are capable of handling diverse users and their devices without complicated calibrations or transformations. Experiments using heterogeneous devices in two sites that have different environments have confirmed that our novel scheme is reliable and feasible and achieve better performance than localization algorithms using RSS absolute values. We will then expand the scale of our experiments to cover the entire university and perform long-term usability testing.

\section{Competing Interests}

The authors declare that there are no competing interests regarding the publication of this paper. 


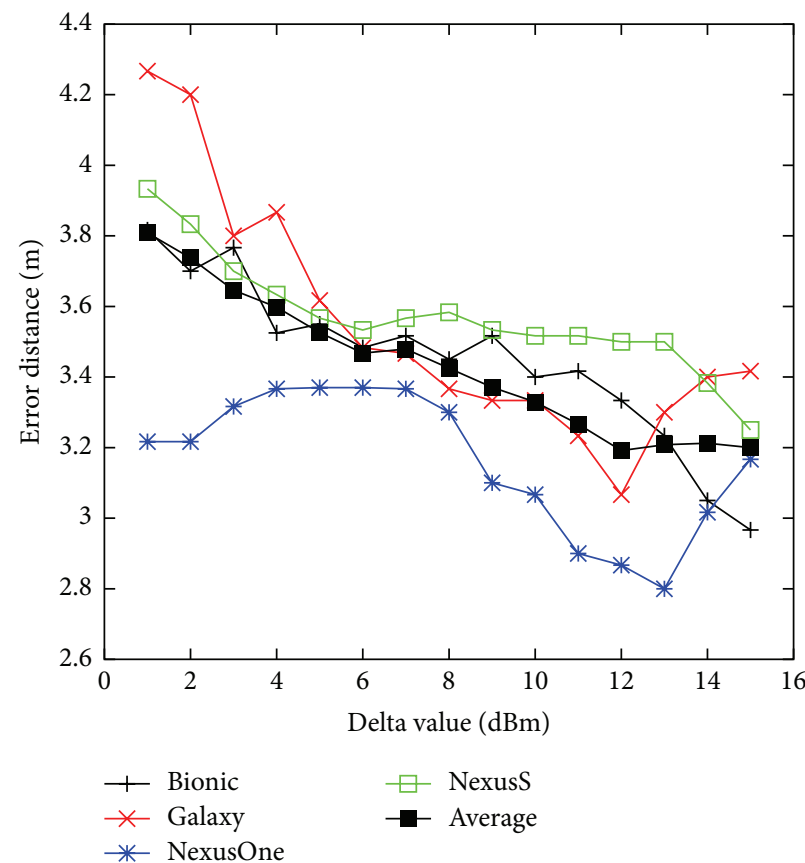

(a) Instant average

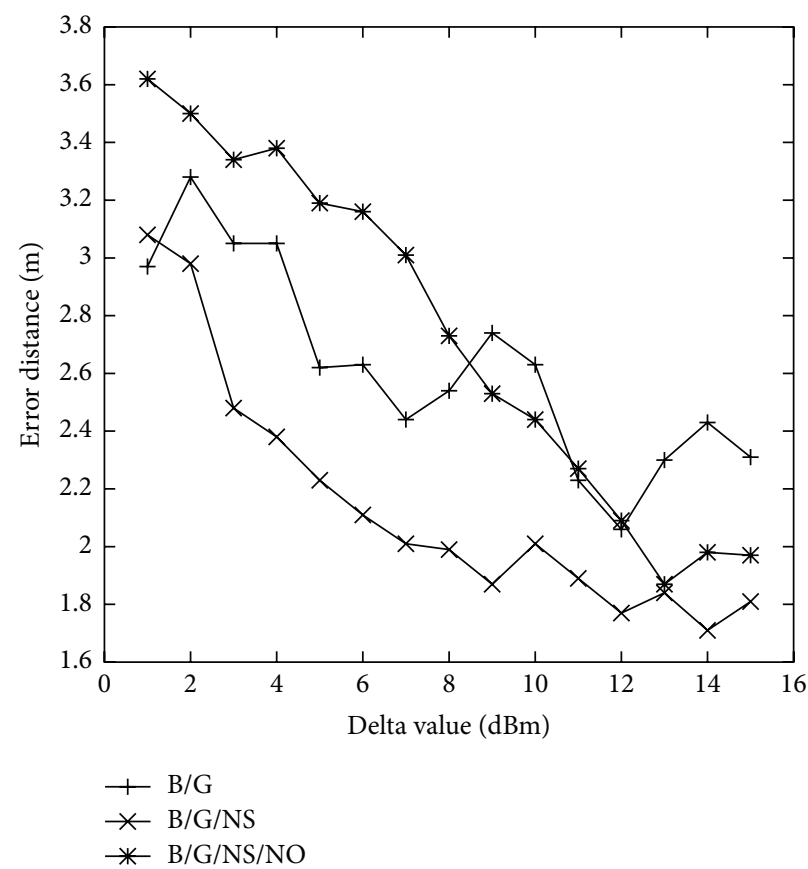

(b) Accumulated average

FIGURE 15: $\delta$ derivation using instant or accumulated fingerprints at UCLA laboratory.

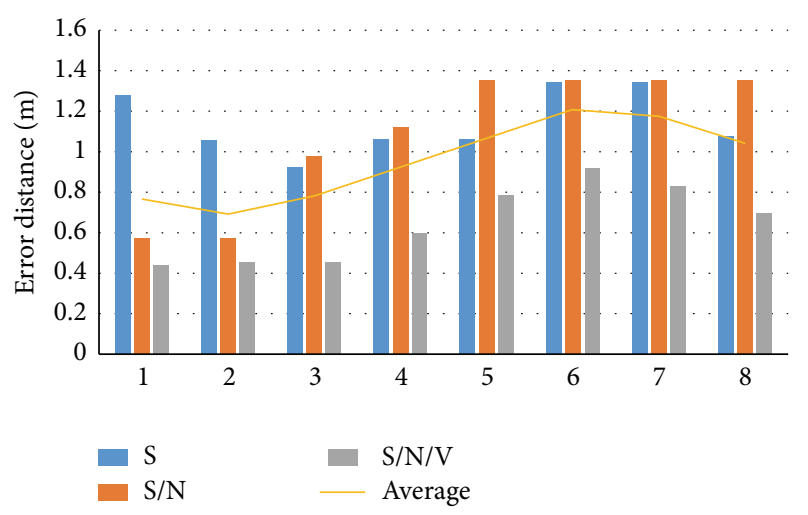

FIGURE 16: Localization performance of a Desire HD fingerprint at 5th floor of the Gachon IT building with varying delta value.

\section{Acknowledgments}

This research was supported by the Gachon University Research Fund GCU-2015-0044 of 2015.

\section{References}

[1] S. Wang, J. Min, and B. K. Yi, "Location based services for mobiles: technologies and standards," Electronics, pp. 78-92, 2008.

[2] D. Mohapatra and S. B. Suma, "Survey of location based wireless services," in Proceedings of the 7th IEEE International Conference on Personal Wireless Communications (ICPWC '05), pp. 358362, January 2005.
[3] M. Mohammadi, E. Molaei, and A. Naserasadi, "A survey on location based services and positioning techniques," International Journal of Computer Applications, vol. 24, no. 5, pp. 1-5, 2011.

[4] Y. Gu, A. Lo, and I. Niemegeers, "A survey of indoor positioning systems for wireless personal networks," IEEE Communications Surveys and Tutorials, vol. 11, no. 1, pp. 13-32, 2009.

[5] V. Honkavirta, T. Perälä, S. Ali-Löytty, and R. Piché, "A comparative survey of WLAN location fingerprinting methods," in Proceedings of the 6th Workshop on Positioning, Navigation and Communication, (WPNC '09), pp. 243-251, Hannover, Germany, March 2009.

[6] D. Zhang, F. Xia, Z. Yang, L. Yao, and W. Zhao, "Localization technologies for indoor human tracking," in Proceedings of the 5th International Conference on Future Information Technology (FutureTech '10), May 2010.

[7] H. Liu, H. Darabi, P. Banerjee, and J. Liu, "Survey of wireless indoor positioning techniques and systems," IEEE Transactions on Systems, Man and Cybernetics Part C: Applications and Reviews, vol. 37, no. 6, pp. 1067-1080, 2007.

[8] N. B. Priyantha, A. Chakraborty, and H. Balakrishnan, "Cricket location-support system," in Proceedings of the 6th Annual International Conference on Mobile Computing and Networking (MOBICOM '00), pp. 32-43, August 2000.

[9] R. Want, A. Hopper, V. Falcão, and J. Gibbons, "The active badge location system," ACM Transactions on Information Systems, vol. 10, no. 1, pp. 91-102, 1992.

[10] P. Bahl and V. N. Padmanabhan, "RADAR: an in-building RFbased user location and tracking system," in Proceedings of the 19th Annual Joint Conference of the IEEE Computer and Communications Societies (INFOCOM '00), pp. 775-784, March 2000 . 
[11] H. Wang, S. Sen, A. Elgohary, M. Farid, M. Youssef, and R. R. Choudhury, "No need to war-drive: unsupervised indoor localization," in Proceedings of the ACM 10th International Conference on Mobile Systems, Applications (MobiSys '12), pp. 197-210, Ambleside, UK, June 2012.

[12] G. Shen, Z. Chen, P. Zhang, T. Moscibroda, and Y. Zhang, "Walkiemarkie: indoor pathway mapping made easy," in Proceedings of the 10th USENIX Conference on Networked Systems Design and Implementation, pp. 85-98, USENIX Association, 2013.

[13] A. LaMarca, Y. Chawathe, S. Consolvo et al., "Place Lab: device positioning using radio beacons in the wild," in Pervasive Computing: Third International Conference, PERVASIVE 2005, Munich, Germany, May 8-13, 2005. Proceedings, vol. 3468 of Lecture Notes in Computer Science, pp. 116-133, Springer Berlin Heidelberg, Berlin, Heidelberg, 2005.

[14] P. Bolliger, "Redpin-adaptive, zero-configuration indoor localization through user collaboration," in Proceedings of the 1st ACM International Workshop on Mobile Entity Localization and Tracking in GPS-Less Environments (MELT '08), pp. 55-60, ACM, San Francisco, Calif, USA, 2008.

[15] M. Lee, H. Yang, D. Han, and C. Yu, "Crowdsourced radiomap for roomlevel place recognition in urban environment," in Proceedings of the 8th IEEE International Conference on Pervasive Computing and Communications Workshops (PERCOM Workshops '10), pp. 648-653, IEEE, Mannheim, Germany, April 2010.

[16] S. Teller, J. Battat, B. Charrow et al., "Organic indoor location discovery," Tech. Rep., MIT, 2008.

[17] J.-G. Park, D. Curtis, S. Teller, and J. Ledlie, "Implications of device diversity for organic localization," in Proceedings of the 30th IEEE International Conference on Computer Communications (INFOCOM '11), pp. 3182-3190, Shanghai, China, April 2011.

[18] J.-G. Park, B. Charrow, D. Curtis et al., "Growing an organic indoor location system," in Proceedings of the 8th International Conference on Mobile Systems, Applications, and Services (MobiSys '10), San Francisco, Calif, USA, June 2010.

[19] J. Ledlie, J. geun Park, D. Curtis, A. Cavalcante, and L. Camara, "Mole: a scalable, user-generated wifi positioning engin," in Proceedings of the International Conference on Indoor Positioning and Indoor Navigation (IPIN '11), pp. 1-10, IEEE, Guimaraes, Portugal, 2011.

[20] A. K. M. Hossain and W.-S. Soh, "A survey of calibration-free indoor positioning systems," Computer Communications, vol. 66, pp. 1-13, 2015.

[21] C. Wu, Z. Yang, Y. Liu, and W. Xi, "WILL: wireless indoor localization without site survey," IEEE Transactions on Parallel and Distributed Systems, vol. 24, no. 4, pp. 839-848, 2013.

[22] A. K. M. M. Hossain, Y. Jin, W.-S. Soh, and H. N. Van, "SSD: a robust RF location fingerprint addressing mobile devices' heterogeneity," IEEE Transactions on Mobile Computing, vol. 12, no. 1, pp. 65-77, 2013.

[23] F. Dong, Y. Chen, J. Liu, Q. Ning, and S. Piao, "A calibrationfree localization solution for handling signal strength variance," in Mobile Entity Localization and Tracking in GPS-Less Environnments: Second International Workshop, MELT 2009, Orlando, FL, USA, September 30, 2009. Proceedings, pp. 79-90, Springer, Berlin, Germany, 2009.

[24] S. Yang, P. Dessai, M. Verma, and M. Gerla, "FreeLoc: calibration-free crowdsourced indoor localization," in Proceedings of the 32nd IEEE Conference on Computer Communications (INFOCOM '13), pp. 2481-2489, Turin, Italy, April 2013.
[25] A. Harter, A. Hopper, P. Steggles, A. Ward, and P. Webster, “The anatomy of a context-aware application," in Proceedings of the 5th Annual ACM/IEEE International Conference on Mobile Computing and Networking (MobiCom '99), pp. 59-68, ACM, 1999.

[26] N. B. Priyantha, A. K. Miu, H. Balakrishnan, and S. Teller, “The cricket compass for context-aware mobile applications," in Proceedings of the International Conference on Mobile Computing and Networking (MobiCom '01), Rome, Italy, July 2001.

[27] L. Ni, Y. Liu, Y. C. Lau, and A. Patil, "Landmarc: indoor location sensing using active rfid," in Proceedings of the 1st IEEE International Conference on Pervasive Computing and Communications (PerCom '03), 10.1109/PERCOM.2003.1192765, pp. 407415, Fort Worth, Tex, USA, March 2003.

[28] G.-Y. Jin, X.-Y. Lu, and M.-S. Park, "An indoor localization mechanism using active RFID tag," in Proceedings of the IEEE International Conference on Sensor Networks, Ubiquitous, and Trustworthy Computing, vol. 1, pp. 40-43, IEEE, Taichung, Taiwan, June 2006.

[29] M. Kanaan and K. Pahlavan, "A comparison of wireless geolocation algorithms in the indoor environment," in Proceedings of the in Wireless Communications and Networking Conference (WCNC '04), vol. 1, pp. 177-182, IEEE, 2004.

[30] K. Kaemarungsi and P. Krishnamurthy, "Properties of indoor received signal strength for WLAN location fingerprinting," in Proceedings of the 1st Annual International Conference on Mobile and Ubiquitous Systems: Networking and Services (MOBIQUITOUS '04), pp. 14-23, August 2004.

[31] S.-H. Fang, T.-N. Lin, and K.-C. Lee, "A novel algorithm for multipath fingerprinting in indoor WLAN environments," IEEE Transactions on Wireless Communications, vol. 7, no. 9, pp. 35793588, 2008.

[32] A. Haeberlen, E. Flannery, A. M. Ladd, A. Rudys, D. S. Wallach, and L. E. Kavraki, "Practical robust localization over large-scale 802.11 wireless networks," in Proceedings of the 10th Annual International Conference on Mobile Computing and Networking (MobiCom '04), pp. 70-84, Philadelphia, Pa, USA, October 2004.

[33] A. W. Tsui, Y.-H. Chuang, and H.-H. Chu, "Unsupervised learning for solving RSS hardware variance problem in WiFi localization," Mobile Networks and Applications, vol. 14, no. 5, pp. 677691, 2009.

[34] D. H. Kim, Y. Kim, D. Estrin, and M. B. Srivastava, "SensLoc: sensing everyday places and paths using less energy," in Proceedings of the 8th ACM International Conference on Embedded Networked Sensor Systems (SenSys '10), pp. 43-56, ACM, Zurich, Switzerland, November 2010.

[35] Y. Jiang, X. Pan, K. Li et al., "ARIEL: automatic Wi-Fi based room fingerprinting for indoor localization," in Proceedings of the 14th International Conference on Ubiquitous Computing (UbiComp '12), pp. 441-450, ACM, Pittsburgh, Pa, USA, September 2012.

[36] K. Pahlavan and P. Krishnamurthy, Principles of Wireless Networks: A Unified Approach, Prentice Hall, New York, NY, USA, 1st edition, 2001.

[37] A. Smailagic, J. Small, and D. P. Siewiorek, "Determining user location for context aware computing through the use of a wireless LAN infrastructure," ACM Mobile Networks and Applications, vol. 6, pp. 1-8, 2000. 

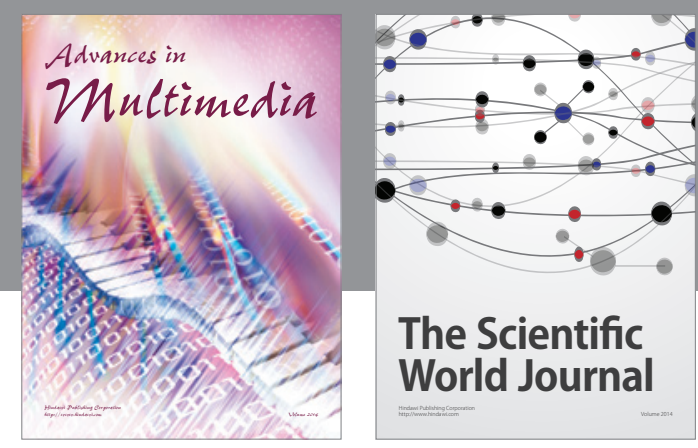

The Scientific World Journal
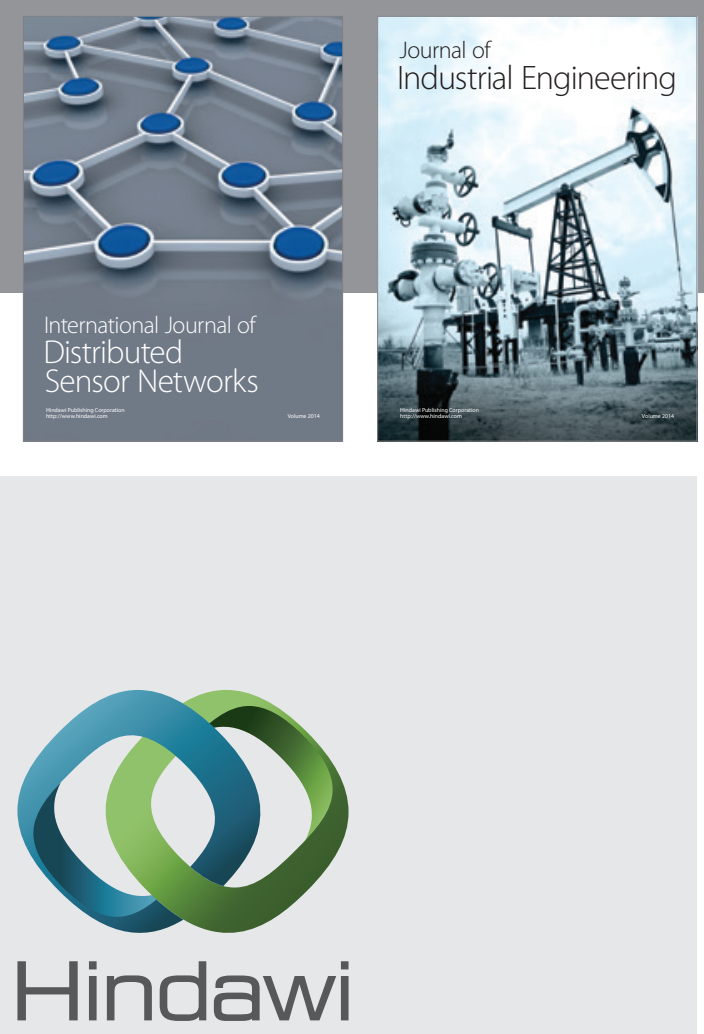

Submit your manuscripts at

http://www.hindawi.com

\section{Computer Networks} and Communications
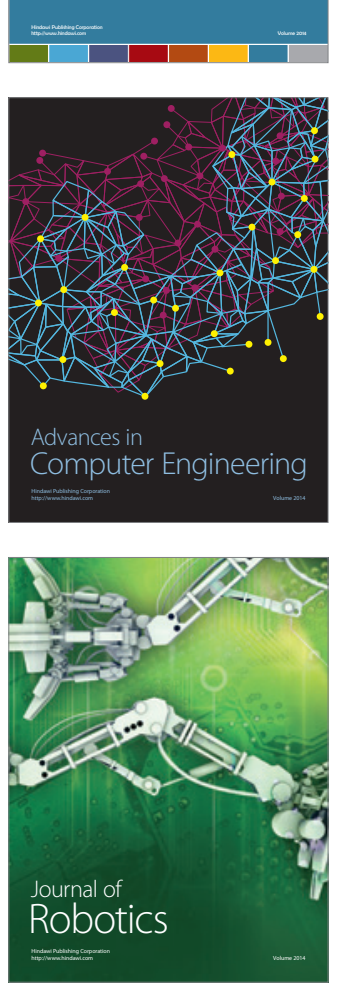
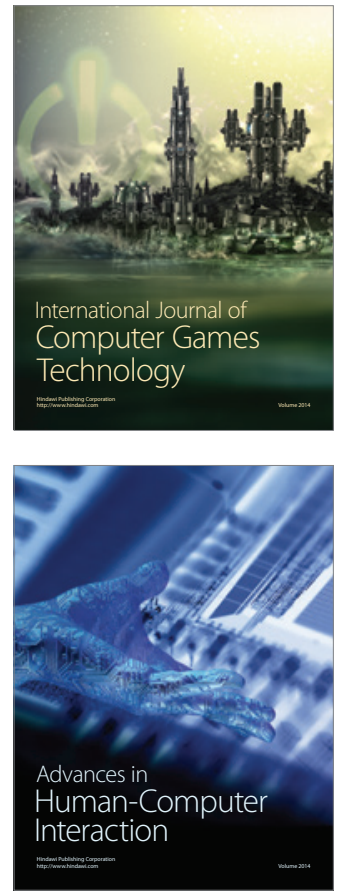
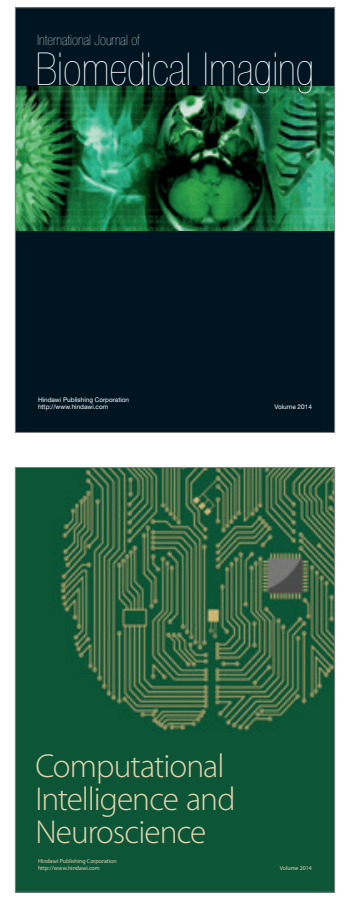
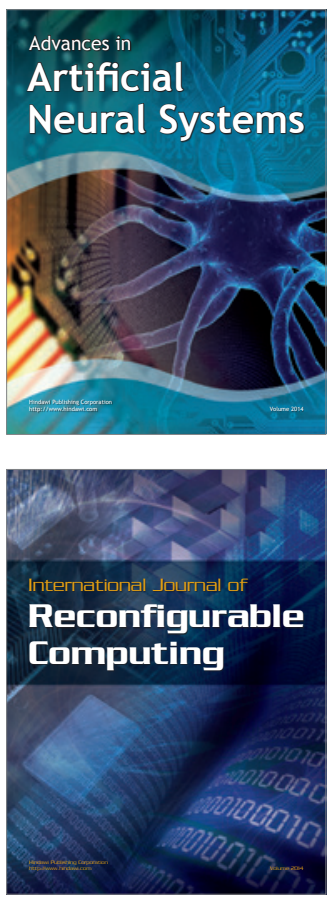
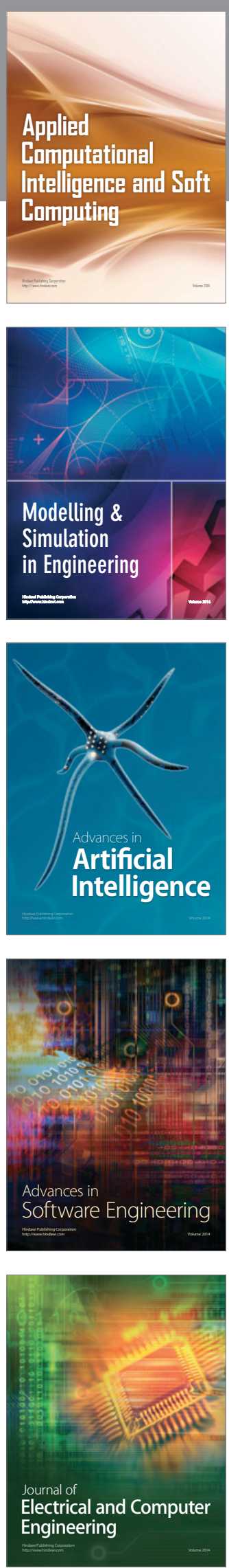